\title{
A Probabilistic Account of Logical Metonymy
}

\author{
Maria Lapata* \\ University of Sheffield
}

\author{
Alex Lascarides ${ }^{\dagger}$ \\ University of Edinburgh
}

In this article we investigate logical metonymy, that is, constructions in which the argument of a word in syntax appears to be different from that argument in logical form (e.g., enjoy the book means enjoy reading the book, and easy problem means a problem that is easy to solve). The systematic variation in the interpretation of such constructions suggests a rich and complex theory of composition on the syntax/semantics interface. Linguistic accounts of logical metonymy typically fail to describe exhaustively all the possible interpretations, or they don't rank those interpretations in terms of their likelihood. In view of this, we acquire the meanings of metonymic verbs and adjectives from a large corpus and propose a probabilistic model that provides a ranking on the set of possible interpretations. We identify the interpretations automatically by exploiting the consistent correspondences between surface syntactic cues and meaning. We evaluate our results against paraphrase judgments elicited experimentally from humans and show that the model's ranking of meanings correlates reliably with human intuitions.

\section{Introduction}

Much work in lexical semantics has been concerned with accounting for regular polysemy, that is, the regular and predictable sense alternations to which certain classes of words are subject (Apresjan 1973). It has been argued that in some cases, the different interpretations of these words must arise from the interaction between the semantics of the words during syntactic composition, rather than by exhaustively listing all the possible senses of a word in distinct lexical entries (Pustejovsky 1991). The class of phenomena that Pustejovsky $(1991,1995)$ has called logical metonymy is one such example. In the case of logical metonymy additional meaning arises for particular verb-noun and adjective-noun combinations in a systematic way: the verb (or adjective) semantically selects for an event-type argument, which is a different semantic type from that denoted by the noun. Nevertheless, the value of this event is predictable from the semantics of the noun. An example of verbal logical metonymy is given in (1) and (2): (1a) usually means (1b) and (2a) usually means (2b).

(1) a. Mary finished the cigarette.

b. Mary finished smoking the cigarette.

(2) a. Mary finished her beer.

b. Mary finished drinking her beer.

* Department of Computer Science, University of Sheffield, Regent Court, 211 Portobello Street, Sheffield S1 4DP, UK. E-mail: mlap@dcs.shef.ac.uk.

$\dagger$ School of Informatics, University of Edinburgh, 2 Buccleuch Place, Edinburgh EH8 9LW, UK. E-mail: alex@inf.ed.ac.uk. 
Note how the events in these examples correspond to the purpose of the object denoted by the noun: the purpose of a cigarette is to smoke it and the purpose of a beer is to drink it. Similarly, (3a) means a problem that is easy to solve, (3b) means a language that is difficult to learn, speak, or write, (3c) means a cook that cooks well, (3d) means a soup that tastes good, (3e) means someone who programmes fast, and (3f) means a plane that flies quickly.

(3) a. easy problem

b. difficult language

c. good cook

d. good soup

e. fast programmer

f. fast plane

The interpretations of logical metonymies can typically be rendered with a paraphrase, as we have indicated for the above examples. Verb-nouns are paraphrased with a progressive or infinitive VP that is the complement of the polysemous verb (e.g., smoking in (1b)) and whose object is the NP figuring in the verb-noun combination (e.g., cigarette in (1b)). Adjective-noun combinations are usually paraphrased with a verb modified by the adjective in question or its corresponding adverb. For example, an easy problem is a problem that is easy to solve or a problem that one can solve easily (see (3a)).

Logical metonymy has been extensively studied in the lexical semantics literature. Previous approaches have focused on descriptive (Vendler 1968) or theoretical (Pustejovsky 1991, 1995; Briscoe, Copestake, and Boguraev 1990) accounts, on the linguistic constraints on the phenomenon (Godard and Jayez 1993; Pustejovsky and Bouillon 1995; Copestake and Briscoe 1995; Copestake 2001), and on the influence of discourse context on the interpretation of metonymies (Briscoe, Copestake, and Boguraev 1990; Lascarides and Copestake 1998; Verspoor 1997). McElree et al. (2001) investigated the on-line processing of metonymic expressions; their results indicate that humans display longer reading times for sentences like (1a) than for sentences like (1b).

There are at least two challenges in providing an adequate account of logical metonymy. The first concerns semi-productivity: There is a wealth of evidence that metonymic constructions are partially conventionalized, and so resolving metonymy entirely via pragmatic reasoning (e.g., by computing the purpose of the object that is denoted by the noun according to real-world knowledge) will overgenerate the possible interpretations (Hobbs et al. 1993). For example, the logical metonymies in (4) are odd, even though pragmatics suggests an interpretation (because real-world knowledge assigns a purpose to the object denoted by the NP):

(4) a. ?John enjoyed the dictionary.

b. ?John enjoyed the door.

c. ?John began/enjoyed the highway.

d. ?John began the bridge.

Sentence (4a) is odd because the purpose of dictionaries is to refer to them, or to consult them. These are (pointlike) achievements and cannot easily combine with enjoy, which has to be true of an event with significant duration. Domain knowledge assigns doors, highways, and bridges a particular purpose, and so the fact that the sentences in $(4 \mathrm{~b})-(4 \mathrm{~d})$ are odd indicates that metonymic interpretations are subject to conventional constraints (Godard and Jayez 1993). 
The second challenge concerns the diversity of possible interpretations of metonymic constructions. This diversity is attested across and within metonymic constructions. Metonymic verbs and adjectives are able to take on different meanings depending on their local context, namely, the noun or noun class they select as objects (in the case of verbs) or modify (in the case of adjectives). Consider the examples in (1), in which the meaning of the verb finish varies depending on the object it selects. Similarly, the adjective good receives different interpretations when modifying the nouns cook and soup (see (3c) and (3d)).

Although we've observed that some logical metonymies are odd even though pragmatics suggests an interpretation (e.g., (4c)), Vendler (1968) acknowledges that other logical metonymies have more than one plausible interpretation. In order to account for the meaning of adjective-noun combinations, Vendler (1968, page 92) points out that "in most cases not one verb, but a family of verbs is needed". For example, fast scientist can mean a scientist who does experiments quickly, publishes quickly, and so on.

Vendler (1968) further observes that the noun figuring in an adjective-noun combination is usually the subject or object of the paraphrasing verb. Although fast usually triggers a verb-subject interpretation (see (3e) and (3f)), easy and difficult trigger verbobject interpretations (see (3a) and (3b)). An easy problem is usually a problem that one solves easily (so problem is the object of solve), and a difficult language is a language that one learns, speaks, or writes with difficulty (so language is the object of learn, speak, and write). Adjectives like good allow either verb-subject or verb-object interpretations: a good cook is a cook who cooks well, whereas good soup is a soup that tastes good.

All of these interpretations of fast scientist, difficult language, or good soup seem highly plausible out of context, though one interpretation may be favored over another in a particular context. In fact, in sufficiently rich contexts, pragmatics can even override conventional interpretations: Lascarides and Copestake (1998) suggest that (5c) means $(5 d)$ and not (5e):

(5) a. All the office personnel took part in the company sports day last week.

b. One of the programmers was a good athlete, but the other was struggling to finish the courses.

c. The fast programmer came first in the $100 \mathrm{~m}$.

d. The programmer who runs fast came first in the $100 \mathrm{~m}$.

e. The programmer who programs fast came first in the $100 \mathrm{~m}$.

The discourse context can also ameliorate highly marked logical metonymies, such as $(4 \mathrm{c})$ :

(6) a. John uses two highways to get to work every morning.

b. He first takes H-280 and then H-101.

c. He always enjoys H-280,

d. but the traffic jams on $\mathrm{H}-101$ frustrate him.

Arguably the most influential account of logical metonymy is Pustejovsky's (1991, 1995) theory of the generative lexicon. Pustejovsky avoids enumerating the various senses for adjectives like fast and verbs like finish by exploiting a rich lexical semantics for nouns. The lexical entry for an artifact-denoting noun includes a qualia structure: this specifies key features of the word's meaning that are in some sense 
derivable from real-world knowledge but are lexicalized so as to influence conventional processes. The qualia structure includes a telic role (i.e., the purpose of the object denoted by the noun) and an agentive role (i.e., the event that brought the object into existence). Thus the lexical entry for book includes a telic role with a value equivalent to read and an agentive role with a value equivalent to write, whereas for cigarette the telic role is equivalent to smoke and the agentive role is equivalent to roll or manufacture.

When finish combines with an object-denoting NP, a metonymic interpretation is constructed in which the missing information is provided by the qualia structure of the NP. More technically, semantic composition of finish with cigarette causes the semantic type of the noun to be coerced into its telic event (or its agentive event), and the semantic relation corresponding to the metonymic verb (finish) predicates over this event. This results in an interpretation of (1a) equivalent to (1b). Verbs like begin and enjoy behave in a similar way. Enjoy the book can mean enjoy reading the book, because of book's telic role, or enjoy writing the book, because of book's agentive role. In fact, the agentive reading is less typical for book than the telic one, but for other nouns the opposite is true. For instance, begin the tunnel can mean begin building the tunnel, but the interpretation that is equivalent to begin going through the tunnel is highly marked. There is also variation in the relative likelihood of interpretations among different metonymic verbs. (We will return to this issue shortly.) The adjectivenoun combinations are treated along similar lines. Thus the logical polysemy of words like finish and fast is not accounted for by exhaustive listing. ${ }^{1}$

In contrast to the volume of theoretical work on logical metonymy, very little empirical work has tackled the topic. Briscoe et al. (1990) investigate the presence of verbal logical metonymies in naturally occurring text by looking into data extracted from the Lancaster-Oslo/Bergen corpus (LOB, one million words). Verspoor (1997) undertakes a similar study in the British National Corpus (BNC, 100 million words). Both studies investigate how widespread the use of logical metonymy is, and how far the interpretation for metonymic examples can be recovered from the head noun's qualia structure, assuming one knows what the qualia structure for any given noun is. Neither of these studies is concerned with the automatic generation of interpretations for logical metonymies and the determination of their likelihood.

Although conceptually elegant, Pustejovsky's (1995) theory of the generative lexicon does not aim to provide an exhaustive description of the telic roles that a given noun may have. However, these roles are crucial for interpreting verb-noun and adjective-noun metonymies. In contrast to Vendler (1968), who acknowledges that logical metonymies may trigger more than one interpretation (in other words, that there may be more than one possible event associated with the noun in question), Pustejovsky implicitly assumes that nouns or noun classes have one (perhaps default) telic role without, however, systematically investigating the relative degree of ambiguity of the various cases of logical metonymy (e.g., the out-of-context possible readings for fast scientist suggest that fast scientist exhibits a higher degree of semantic ambiguity than fast plane). One could conceivably represent this by the generality of the semantic type of the telic role in the various nouns (e.g., assign the telic role of scientist a relatively general type of event compared with that for plane). But this simply transfers the problem: The degree of generality in lexical representation is highly idiosyncratic and ideally should be acquired from linguistic evidence; furthermore, for nouns with

\footnotetext{
1 Other lexical accounts, such as Copestake and Briscoe (1995), differ from Pustejovsky's (1995) in that the coercion is treated as internal to the semantics of the metonymic verb or adjective rather than the noun; motivation for this comes from copredication data, such as the acceptability of fast and intelligent typist and John picked up and finished his beer.
} 
a general telic role, pragmatics would have to do 'more work' to augment the general interpretation with a more specific one. Even in theories in which more than one interpretation is provided (see Vendler 1968), no information is given with respect to the relative likelihood of these interpretations.

Pustejovsky's account also doesn't predict the degree of variation of interpretations for a given noun among the different metonymic verbs: for example, the fact that begin the house is, intuitively at least, more likely to resolve to an agentive-role interpretation (i.e., begin building the house) than a telic-role interpretation (i.e., begin living in the house), whereas the reverse is true of enjoy the house. Ideally, we would like a model of logical metonymy that reflects this variation in interpretation.

In this article we aim to complement the theoretical work on the interpretation of logical metonymy by addressing the following questions: (1) Can the meanings of metonymic adjective-noun and verb-noun combinations be acquired automatically from corpora? (2) Can we constrain the number of interpretations of these combinations by providing a ranking on the set of possible meanings? (3) Can we determine whether a particular adjective has a preference for a verb-subject or a verb-object interpretation? We provide a probabilistic model that uses distributional information extracted from a large corpus to interpret logical metonymies automatically without recourse to pre-existing taxonomies or manually annotated data.

The differences among the various theoretical accounts-for example, that Copestake and Briscoe (1995) treat type coercion as internal to the metonymic word, whereas Pustejovsky (1995) treats it as part of the noun-do not matter for our purposes, because we aim to provide information about metonymic interpretations that is compatible with either account. More specifically, we are concerned with using a real-language corpus to acquire automatically the semantic value of the event that is part of the interpretation. We abstract away from theoretical concepts such as semantic type coercion and instead utilize co-occurrence frequencies in the corpus to predict metonymic interpretations. Very roughly, we acquire a ranked set of interpretations enjoy $V$-ing the book for the construction enjoy the book by estimating the probabilities that $\mathrm{V}$ is enjoyed and that it is something done to books; and we estimate these probabilities on the basis of the corpus frequencies for V's appearing as a (verbal) complement to enjoy and for V's taking book as its object. Similarly, we acquire a ranked set of verb-subject interpretations of fast plane by estimating the likelihood of seeing the plane Vs and Vs quickly in the corpus. (See Sections 2 and 3 for more details and motivation of these models.)

Our results show not only that we can predict meaning differences when the same adjective or verb is associated with different nouns, but also that we can derive-taking into account Vendler's (1968) observation-a cluster of meanings for a single verb- or adjective-noun combination. We can also predict meaning differences for a given noun associated with different metonymic verbs and adjectives. We evaluate our results by comparing the model's predictions to human judgments and show that the model's ranking of meanings correlates reliably with human intuitions.

However, the model is limited in its scope. It is suited for the interpretation of wellformed metonymic constructions. But it does not distinguish odd metonymies (see (4)) from acceptable ones: in both cases, paraphrases will be generated, at least in principle (see Section 2.1 for explanation and motivation). In particular, the model does not learn conventional constraints, such as that enjoy must take an event of duration as its argument (Godard and Jayez 1993). However, such constraints are potentially captured indirectly: If the above conventional constraint is right, enjoy referring to should not be attested in the corpus, and hence according to our model it won't be part of a possible paraphrase for enjoy the dictionary (see Sections 2.4.2 and 2.5.3 for further discussion). Further, since the model abstracts away from semantic type coercion, it 
does not distinguish between uses of a verb or adjective that are claimed in the lexical semantics literature to be metonymic uses (e.g., enjoy the book, fast programmer) and those that are claimed to be nonmetonymic uses (e.g., enjoy the marriage, fast run-time). Again, the model of interpretation presented here will generate paraphrases for all these cases (e.g., it will paraphrase enjoy the marriage as enjoy going to or participating in the marriage and fast run-time as run-time that goes by or passes quickly). The model also does not take discourse context into account; for example, it will not predict the intuitive interpretation of (5e). Rather, it determines the most dominant meanings for a given metonymic construction overall, across all of the instances of it in the corpus.

The remainder of this article is organized as follows: in the first part (Section 2) we present our probabilistic model of verbal logical metonymy and describe the model parameters. In Experiment 1 we use the model to derive the meaning paraphrases for verb-noun combinations randomly selected from the BNC (see Section 2.3) and formally evaluate our results against human intuitions. Experiment 2 demonstrates that when compared against human judgments, our model outperforms a naive baseline in deriving a preference ordering for the meanings of verb-noun combinations, and Experiment 3 evaluates an extension of the basic model. In the second part (Section 3), we focus on adjectival logical metonymy. Section 3.1 introduces our probabilistic formalization for polysemous metonymic adjectives and Sections 3.3-3.6 present our experiments and evaluate our results. Overall, the automatically acquired model of logical metonymy reliably correlates with human intuitions and also predicts the relative degree of ambiguity and acceptability of the various metonymic constructions. In Section 4 we discuss our results, we review related work in Section 5, and we conclude in Section 6.

\section{Metonymic Verbs}

\subsection{The Model}

Consider the verb-noun combinations in (7) and (8). Our task is to come up with $(7 b)$ and $(8 b)$ as appropriate interpretations for $(7 a)$ and (8a). Although the interpretations of (7a) and (8a) are relatively straightforward for English speakers, given their general knowledge about coffees and films and the activities or events associated with them, a probabilistic model requires detailed information about words and their interdependencies in order to generate the right interpretation. Examples of such interdependencies are verbs co-occurring with coffee (e.g., drink, make, prepare) or verbs that are related to begin (e.g., make, realize, understand).

(7) a. John began the coffee.

b. John began drinking the coffee.

(8) a. Mary enjoyed the film.

b. Mary enjoyed watching the film.

A relatively straightforward approach to the interpretation of (7a) and (8a) would be to extract from the corpus (via parsing) paraphrases in which the additional information (e.g., drinking and watching), which is absent from (7a) and (8a), is fleshed out. In other words, we would like to find in the corpus sentences whose main verb is begin followed either by the progressive VP complement drinking or by the infinitive to drink, selecting for the NP coffee as its object. In the general case we would like to find the activities or events related both to the verb begin and the noun coffee (e.g., drinking, buying, making, preparing). Similarly, in order to paraphrase (8a) we need information 
about the VP complements that are associated with enjoy and can take film as their object (e.g., watching, making, shooting).

The above paraphrase-based model is attractive given its simplicity: All we need to do is count the co-occurrences of a verb, its complements, and their objects. The approach is unsupervised, no manual annotation is required, and no corpus-external resources are used. Such a model relies on the assumption that the interpretations of (7a) and (8a) can be approximated by their usage; that is, it assumes that the likelihood of uttering the metonymic construction is equal to that of uttering its interpretation. However, this assumption is not borne out. Only four sentences in the BNC are relevant for the interpretation of begin coffee (see (9)); likewise, four sentences are relevant for the interpretation of enjoy film (see (10)).

(9) a. Siegfried bustled in, muttered a greeting and began to pour his coffee.

b. She began to pour coffee.

c. Jenna began to serve the coffee.

d. Victor began dispensing coffee.

(10) a. I was given a good speaking part and enjoyed making the film.

b. He's enjoying making the film.

c. Courtenay enjoyed making the film.

d. I enjoy most music and enjoy watching good films.

e. Did you enjoy acting alongside Marlon Brando in the recent film The Freshman?

The attested sentences in (9) are misleading if they are taken as the only evidence for the interpretation of begin coffee, for on their own they suggest that the most likely interpretation for begin coffee is begin to pour coffee, whereas begin to serve coffee and begin dispensing coffee are less likely, as they are attested in the corpus only once. Note that the sentences in (9) fail to capture begin to drink coffee as a potential interpretation for begin coffee. On the basis of the sentences in (10), enjoy making the film is the most likely interpretation for (8a), whereas enjoy watching the film and enjoy acting in the film are equally likely.

This finding complies with Briscoe, Copestake, and Boguraev's (1990) results for the LOB corpus: begin V NP is very rare when the value of V corresponds to a highly plausible interpretation of begin NP. Indeed, one can predict that problems with finding evidence for begin V NP will occur on the basis of Gricean principles of language production, where the heuristic be brief (which is part of the maxim of manner) will compel speakers to utter begin coffee as opposed to begin $\mathrm{V}$ coffee if $\mathrm{V}$ is one of the plausible interpretations of begin coffee. Thus on the basis of this Gricean reasoning, one might expect metonymies like (7a) and (8a) to occur with greater frequencies than their respective paraphrases (see (7b) and (8b)). Tables 1-3 show BNC counts of verbnoun metonymies (commonly cited in the lexical semantics literature (Pustejovsky 1995; Verspoor 1997)) and their corresponding interpretations when these are attested in the corpus. The data in Tables 1-3 indicate that metonymic expressions are more often attested in the BNC with NP rather than with VP complements.

The discrepancy between an interpretation and its usage could be circumvented by using a corpus labeled explicitly with interpretation paraphrases. Lacking such a corpus, we will sketch below an approach to the interpretation of metonymies that retains the simplicity of the paraphrase-based account but no longer assumes a tight correspondence between a metonymic interpretation and its usage. We present an 
Table 1

BNC frequencies for begin.

\begin{tabular}{lcc}
\hline Examples & begin $\mathbf{N P}$ & begin $\mathbf{V - i n g}$ NP \\
\hline begin book & 35 & 17 \\
begin sandwich & 4 & 0 \\
begin beer & 2 & 1 \\
begin speech & 21 & 4 \\
begin solo & 1 & 1 \\
begin song & 19 & 8 \\
begin story & 31 & 15 \\
\hline
\end{tabular}

Table 2

BNC frequencies for enjoy.

\begin{tabular}{lcc}
\hline Examples & enjoy NP & enjoy $\mathbf{V - i n g ~ N P}$ \\
\hline enjoy symphony & 34 & 30 \\
enjoy movie & 5 & 1 \\
enjoy coffee & 8 & 1 \\
enjoy book & 23 & 9 \\
like movie & 18 & 3 \\
\end{tabular}

Table 3

BNC frequencies for want.

\begin{tabular}{lcc}
\hline Examples & want $\mathbf{N P}$ & want $\mathbf{V}$-ing NP \\
\hline want cigarette & 18 & 3 \\
want beer & 15 & 8 \\
want job & 116 & 60
\end{tabular}

unsupervised method that generates interpretations for verbal metonymies without recourse to manually annotated data or taxonomic information; it requires only a part-of-speech-tagged corpus and a partial parser.

We model the interpretation of a verbal metonymy as the joint distribution $P(e, 0, v)$ of three variables: the metonymic verb $v$ (e.g., enjoy), its object $o$ (e.g., film), and the sought-after interpretation $e$ (e.g., making, watching, directing). By choosing the ordering $\langle e, v, o\rangle$ for the variables $e, v$, and $o$, we can factor $P(e, o, v)$ as follows:

$$
P(e, o, v)=P(e) \cdot P(v \mid e) \cdot P(o \mid e, v)
$$

The probabilities $P(e), P(v \mid e)$, and $P(o \mid e, v)$ can be estimated using maximum likelihood as follows:

$$
\begin{aligned}
\hat{P}(e) & =\frac{f(e)}{N} \\
\hat{P}(v \mid e) & =\frac{f(v, e)}{f(e)} \\
\hat{P}(o \mid e, v) & =\frac{f(o, e, v)}{f(e, v)}
\end{aligned}
$$


Table 4

Most frequent complements of enjoy and film.

\begin{tabular}{lllr}
\hline \multicolumn{1}{c}{$f($ enjoy, $\boldsymbol{e})$} & & \multicolumn{2}{c}{$f($ film, $\boldsymbol{e})$} \\
\hline play & 44 & make & 176 \\
watch & 42 & be & 154 \\
work with & 35 & see & 89 \\
read & 34 & watch & 65 \\
make & 27 & show & 42 \\
see & 24 & produce & 29 \\
meet & 23 & have & 24 \\
go to & 22 & use & 21 \\
use & 17 & do & 20 \\
take & 15 & get & 18
\end{tabular}

Although $P(e)$ and $P(v \mid e)$ can be estimated straightforwardly from a corpus $(f(e)$ amounts to the number of the times a given verb $e$ is attested, $N$ is the number of verbs found in the corpus (excluding modals and auxiliaries), and $P(v \mid e)$ can be obtained through parsing, by counting the number of times a verb $v$ takes $e$ as its complement), the estimation of $P(o \mid e, v)$ is problematic. It presupposes that co-occurrences of metonymic expressions and their interpretations are to be found in a given corpus, but as we've seen previously, there is a discrepancy between a metonymic interpretation and its usage. In fact, metonymies occur more frequently than their overt interpretations (expressed by the term $f(o, e, v)$ in (14)), and the interpretations in question are not explicitly marked in our corpus. We will therefore make the following approximation:

$$
\begin{gathered}
P(o \mid e, v) \approx P(o \mid e) \\
\hat{P}(o \mid e)=\frac{f(o, e)}{f(e)}
\end{gathered}
$$

The rationale behind this approximation is that the likelihood of seeing a noun $o$ as the object of an event $e$ is largely independent of whether $e$ is the complement of another verb. In other words, $v$ is conditionally independent of $e$, since the likelihood of $o$ is (largely) determined on the basis of $e$ and not of $v$. Consider again example (8a): Mary enjoyed the film. Here, film, the object of enjoy, is more closely related to the underspecified interpretation $e$ rather than to enjoy. For example, watching movies is more likely than eating movies, irrespective of whether Mary enjoyed or liked watching them. We estimate $P(o \mid e)$ as shown in (16). The simplification in (15) results in a compact model with a relatively small number of parameters that can be estimated straightforwardly from the corpus in an unsupervised manner. By substituting equations (12), (13), and (16) into (11) and simplifying the relevant terms, (11) can be rewritten as follows:

$$
P(e, o, v)=\frac{f(v, e) \cdot f(o, e)}{N \cdot f(e)}
$$

Assume we want to generate meaning paraphrases for the verb-noun pair enjoy film (see (8a)). Table 4 lists the most frequent events related to the verb enjoy and the most frequent verbs that take film as their object (we describe how the frequencies $f(v, e)$ and $f(o, e)$ were obtained in the following section). We can observe that seeing, watching, making, and using are all events associated with enjoy and with film and will 
be therefore generated as likely paraphrases for the metonymic expression enjoy film (see Table 4, in which the underlined verbs indicate common complements between the metonymic verb and its object).

Note that the model in (17) does not represent the fact that the metonymic verb $v$ may have a subject. This in practice means that the model cannot distinguish between the different readings for (18a) and (18b): in (18a) the doctor enjoyed watching the film, whereas in (18b) the director enjoyed making or directing the film. The model in (17) will generate the set of events that are associated with enjoying films (e.g., watching, making, seeing, going to), ignoring the contribution of the sentential subject. We present in Section 2.5.1 an extension of the basic model that takes sentential subjects into account.

(18) a. The doctor enjoyed the film.

b. The director enjoyed the film.

It is important to stress that the probabilistic model outlined above is a model of the interpretation rather than the grammaticality of metonymic expressions. In other words, we do not assume that it can distinguish between well-formed and odd metonymic expressions (see the examples in (4)). In fact, it will generally provide a set of interpretation paraphrases, even for odd formulations. The model in (11) has no component that corresponds to the occurrence of $v$ and $o$ together. Choosing the ordering $\langle o, v, e\rangle$ for the variables $o, e$, and $v$ would result in the following derivation for $P(e, o, v)$ :

$$
P(e, o, v)=P(o) \cdot P(v \mid o) \cdot P(e \mid o, v)
$$

The term $P(v \mid o)$ in (19) explicitly takes into account the likelihood of occurrence of the metonymic expression. This means that no interpretation will be provided for odd metonymies like enjoy the highway as long as they are not attested in the corpus. Such a model penalizes, however, well-formed metonymies that are not attested in the corpus. A striking example is enjoy the ice cream, which is a plausible metonymy not attested at all in the BNC and thus by (19) would be incorrectly assigned no interpretations. This is because the maximum-likelihood estimate of $P(v \mid o)$ relies on the co-occurrence frequency $f(v, o)$, which is zero for enjoy the ice cream. But the probabilistic model in (11) will generate meaning paraphrases for metonymic verb-object pairs that have not been attested in the corpus as long as the co-occurrence frequencies $f(v, e)$ and $f(o, e)$ are available.

Finally, note that our model is ignorant with respect to the discourse context within which a given sentence is embedded. This means that it will come up with the same ranked set of meanings for (20b), irrespective of whether it is preceded by sentence (20a) or (21a). The model thus does not focus on the meaning of individual corpus tokens; instead it determines the most dominant meanings for a given verb-noun combination overall, across all of its instances in the corpus.

(20) a. Who is making the cigarettes for tomorrow's party?

b. John finished three cigarettes.

c. John finished making three cigarettes.

(21) a. Why is the room filled with smoke?

b. John finished three cigarettes.

c. John finished smoking three cigarettes. 


\subsection{Parameter Estimation}

We estimated the parameters of the model outlined in the previous section from a partof-speech-tagged and lemmatized version of the BNC, a 100-million-word collection of samples of written and spoken language from a wide range of sources designed to represent current British English (Burnard 1995). The counts $f(v, e)$ and $f(o, e)$ (see (17)) were obtained automatically from a partially parsed version of the BNC created using Cass (Abney 1996), a robust chunk parser designed for the shallow analysis of noisy text. The parser's built-in function was employed to extract tuples of verb-subjects and verb-objects (see (22)). Although verb-subject relations are not relevant for the present model, they are important for capturing the influence of the sentential subject (see Section 2.5) and modeling the interpretations of polysemous adjectives (which we discuss in Section 3).

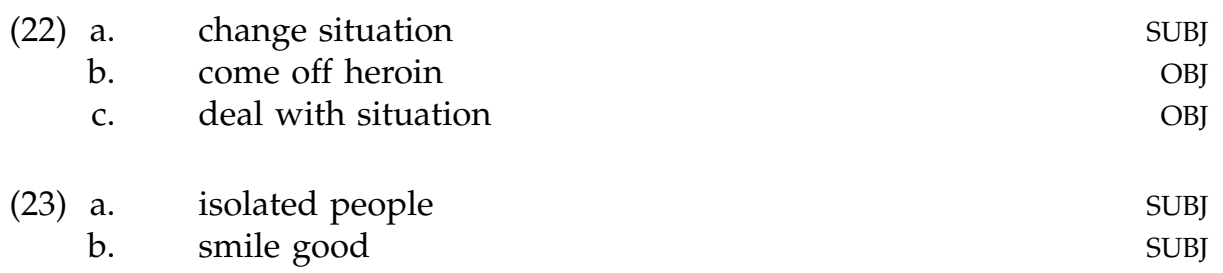

The tuples obtained from the parser's output are an imperfect source of information about argument relations. Bracketing errors, as well as errors in identifying chunk categories accurately, results in tuples whose lexical items do not stand in a verbargument relationship. For example, inspection of the original BNC sentences from which the tuples in (23) were derived reveals that the verb be is missing from (23a) and the noun smile is missing from (23b) (see the sentences in (24)).

(24) a. Wenger found that more than half the childless old people in her study of rural Wales saw a relative, a sibling, niece, nephew or cousin at least once a week, though in inner city London there were more isolated old people.

b. I smiled my best smile down the line.

In order to compile a comprehensive count of verb-argument relations, we discarded tuples containing verbs or nouns attested in a verb-argument relationship only once. Instances of the verb be were also eliminated, since they contribute no semantic information with respect to the events or activities that are possibly associated with the noun with which the verb is combined. Particle verbs (see (22b)) were retained only if the particle was adjacent to the verb. Verbs followed by the preposition by and a head noun were considered instances of verb-subject relations. The verb-object tuples also included prepositional objects (see (22c)). It was assumed that PPs adjacent to the verb headed by any of the prepositions in, to, for, with, on, at, from, of, into, through, and upon were prepositional objects. ${ }^{2}$ This resulted in 737,390 distinct types of verb-subject pairs and 1,078,053 distinct types of verb-object pairs (see Table 5, which presents information about the tuples extracted from the corpus before and after the filtering).

2 The POS tagging of the BNC (Leech, Garside, and Bryant 1994) distinguishes between verb particle constructions like down in climb down the mountain and up in put up the painting, on the one hand, and prepositions, on the other. So this allowed us to distinguish PP complements from NP ones. 
Table 5

Number of tuples extracted from the BNC.

\begin{tabular}{cccrrr}
\hline & \multicolumn{2}{c}{ Tokens } & \multicolumn{3}{c}{ Types } \\
Relation & Parser & Filtering & Tuples & Verbs & Nouns \\
\hline SUBJ & $4,759,950$ & $4,587,762$ & 737,390 & 14,178 & 25,900 \\
OBJ & $3,723,998$ & $3,660,897$ & $1,078,053$ & 12,026 & 35,867
\end{tabular}

The frequency $f(v, e)$ represents verbs taking progressive or infinitive VP complements. These were extracted from the parser's output by looking for verbs followed by progressive or infinitival complements (a special tag, VDG, is reserved in the BNC for verbs in the progressive). The latter were detected by looking for verbs followed by infinitives (indicated by the marker to (TO0) and a verb in base form (VVI)). The examples below illustrate the information extracted from the parser's output for obtaining the frequency $f(v, e)$, which collapsed counts for progressive and infinitive complements.

(25) a. I had started to write a love-story.

b. She started to cook with simplicity.

c. The suspect attempted to run off.

(26) a. I am going to start writing a book.

b. I've really enjoyed working with you.

c. The phones began ringing off the hook. start write

start cook

attempt run off

start write

enjoy work with

begin ring off

Note that some verbs (e.g., start) allow both an infinitival and a progressive complement (see (25a) and (26a), respectively), whereas other verbs (e.g., attempt) allow only one type of complement (see (25c)). Even for verbs that allow both types of complements, there exist syntactic contexts in which the two complement types are in complementary distribution: to start writing occurs 15 times in the BNC, whereas to start to write does not occur at all. The situation is reversed for starting writing and starting to write, for the former does not occur and the latter occurs seven times. Choosing to focus only on one type of complement would result in a lower count for $f(v, e)$ than collapsing the counts observed for both types of complements.

Once we have obtained the frequencies $f(v, e)$ and $f(o, e)$, we can determine the most likely interpretations for metonymic verb-noun combinations. Note that we may choose to impose thresholds on the frequencies $f(v, e)$ and $f(o, e)$ (e.g., $f(v, e)>1$, and $f(o, e)>1)$, depending on the quality of the parsing data or the type of meaning paraphrases we seek to discover (e.g., likely versus unlikely ones).

As an example of the paraphrases generated by our model, consider the sentences in Table 6, which were cited as examples of logical metonymy in the lexical semantics literature (Pustejovsky 1995; Verspoor 1997). The five most likely interpretations for these metonymies (and their respective log-transformed probabilities) are illustrated in Table 7. Note that the model comes up with plausible meanings, some of which overlap with those suggested in the lexical semantics literature (underlined interpretations indicate agreement between the model and the literature). Also, the model derives several meanings, as opposed to the single interpretations provided in most cases in the literature. Consider, for example, the pair begin story in Table 7. Here, not only the interpretation tell is generated, but also write, read, retell, and recount. Another example 
Table 6

Paraphrases for verb-noun combinations taken from the literature.

\begin{tabular}{lll}
\hline John began the story & $\rightarrow$ telling & (Verspoor 1997, page 189) \\
John began the song & $\rightarrow$ singing & (Verspoor 1997, page 189) \\
John began the sandwich & $\rightarrow$ eating/making & (Verspoor 1997, page 167) \\
Mary wants a job & $\rightarrow$ to have & (Pustejovsky 1995, page 45) \\
John began the book & $\rightarrow$ reading/writing & (Verspoor 1997, page 167) \\
Bill enjoyed Steven King's last book & $\rightarrow$ reading & (Pustejovsky 1995, page 88) \\
John began the cigarette & $\rightarrow$ smoking & (Verspoor 1997, page 167) \\
Harry wants another cigarette & $\rightarrow$ to smoke & (Pustejovsky 1995, page 109)
\end{tabular}

Table 7

Model-derived paraphrases for verbal metonymies, ranked in order of likelihood.

\begin{tabular}{llllllll}
\hline \multicolumn{2}{c}{ begin story } & \multicolumn{2}{c}{ begin song } & \multicolumn{2}{c}{ begin sandwich } & \multicolumn{2}{c}{ want job } \\
\hline tell & -16.34 & sing & -15.14 & bite into & -18.12 & get & -14.87 \\
write & -17.02 & rehearse & -16.15 & eat & -18.23 & lose & -15.72 \\
read & -17.28 & write & -16.86 & munch & -19.13 & take & -16.40 \\
retell & -17.45 & hum & -17.45 & unpack & -19.14 & make & -16.52 \\
recount & -17.80 & play & -18.01 & make & -19.42 & create & -16.62
\end{tabular}

\begin{tabular}{lllllllll}
\multicolumn{2}{c}{ begin book } & \multicolumn{2}{c}{ enjoy book } & & \multicolumn{2}{c}{ begin cigarette } & \multicolumn{2}{c}{ want cigarette } \\
\hline read & -15.49 & read & -16.48 & smoke & -16.92 & smoke & -16.67 \\
write & -15.52 & write & -17.58 & roll & -17.63 & take & -18.23 \\
appear in & -16.98 & browse through & -18.56 & light & -17.76 & light & -18.45 \\
publish & -17.10 & look through & -19.68 & take & -18.88 & put & -18.51 \\
leaf through & -17.35 & publish & -19.93 & twitch & -19.17 & buy & -18.64
\end{tabular}

is begin song, for which the model generates the interpretations rehearse, write, hum, and play, in addition to sing.

The model also exhibits slight variation in the interpretations for a given noun among the different metonymic verbs (compare begin book and enjoy book and begin cigarette and want cigarette in Table 7). This is in line with claims made in the lexical semantics literature (Copestake and Briscoe 1995; Pustejovsky 1995; Verspoor 1997), and it ultimately contributes to an improved performance against a "naive baseline" model (see Section 2.4).

In some cases, the model comes up with counterintuitive interpretations: bite into is generated as the most likely interpretation for begin sandwich (although the latter interpretation is not so implausible, since eating entails biting into). The model also fails to rank have as one of the five most likely interpretations for want job (see Table 7). The interpretations get and take are, however, relatively likely; note that they semantically entail the desired interpretation-namely, have-as a poststate. The interpretations make and create imply the act of hiring rather than finding a job. Our model cannot distinguish between the two types of interpretations. It also cannot discover related meanings: for example, that get and take mean have or that tell, retell, and recount (see Table 7) mean tell. (We return to this issue in Section 4.)

In the following section we test our model against verb-noun pairs randomly selected from the BNC and evaluate the meaning paraphrases it generates against human judgments. We explore the linear relationship between the subjects' rankings and the model-derived probabilities using correlation analysis. 


\subsection{Experiment 1: Comparison against Human Judgments}

Although there is no standard way to evaluate the paraphrases generated by the model (there is no gold standard for comparison), a reasonable way to judge the model's performance would seem to be its degree of agreement with human paraphrase ratings. This can be roughly measured by selecting some metonymic constructions, deriving their paraphrase interpretations using the model outlined in Section 2.1, eliciting human judgments on these paraphrases, and then looking at how well the human ratings correlate with the model probabilities for the same paraphrases.

In the following section we describe our method for assembling the set of experimental materials and eliciting human-subject data for the metonymy paraphrasing task. We use correlation analysis to compare the model probabilities against human judgments and explore whether there is a linear relationship between the modelderived likelihood of a given meaning and its perceived plausibility.

In Section 2.4.1 we introduce a naive model of verbal metonymy that does not take the contribution of the metonymic verb into account; metonymic interpretations (i.e., verbs) are simply expressed in terms of their conditional dependence on their objects. We investigate the naive model's performance against the human judgments and the paraphrases generated by our initial model (see Section 2.4).

\subsubsection{Method.}

2.3.1.1 Materials and Design. From the lexical semantics literature (Pustejovsky 1995; Verspoor 1997; McElree et al. 2001) we compiled a list of 20 verbs that allow logical metonymy. From these we randomly selected 12 verbs (attempt, begin, enjoy, finish, expect, postpone, prefer, resist, start, survive, try, and want). The selected verbs ranged in BNC frequency from 10.9 per million to 905.3 per million. Next, we paired each one of them with five nouns randomly selected from the BNC. The nouns had to be attested in the corpus as the object of the verbs in question. Recall that verb-object pairs were identified using Abney's (1996) chunk parser Cass (see Section 2.2 for details). From the retrieved verb-object pairs, we removed all pairs with BNC frequency of one, as we did not want to include verb-noun combinations that were potentially unfamiliar to the subjects. We used the model outlined in Section 2.1 to derive meaning paraphrases for the 60 verb-noun combinations.

Our materials selection procedure abstracts over semantic distinctions that are made in linguistic analyses. For instance, current models of lexical semantics typically assign verbs such as enjoy a nonmetonymic sense when they are combined with NPs that are purely temporal or eventive in nature, as in enjoy the marriage or enjoy the lecture (Copestake and Briscoe 1995; Verspoor 1997). This is largely because a logical form can be constructed in such cases without the use of semantic type coercion; the eventdenoting NP itself is the argument to the predicate enjoy. We did not rule out such nouns from our materials, however, as our evaluation was conducted on randomly selected verb-noun pairs.

More generally, we abstract over several criteria that Verspoor (1997) used in distinguishing metonymic from nonmetonymic uses within the corpus, and we adopt a linguistically naive approach for two reasons. First, whereas Verspoor (1997) could deploy more refined criteria because she was hand-selecting the materials from the corpus and was focusing only on two metonymic verbs (begin and finish), our materials were randomly sampled and covered a wider range of metonymic constructions. And second, paraphrases for nonmetonymic cases (e.g., that enjoy the lecture can be paraphrased as enjoy attending the lecture or enjoy listening to the lecture) may be useful for some potential NLP applications (see the discussion in Section 4.2), since they provide 
Table 8

Number of generated interpretations as frequency cutoff for $f(v, e)$ and $f(o, e)$ is varied.

\begin{tabular}{lcccc}
\hline Verb-noun & $f(v, e) \geq 1$ & $f(v, e) \geq 2$ & $f(v, e) \geq 3$ & $f(v, e) \geq 4$ \\
& $f(o, e) \geq 1$ & $f(o, e) \geq 2$ & $f(o, e) \geq 3$ & $f(n, e) \geq 4$ \\
\hline finish gig & 11 & 4 & 3 & 1 \\
finish novel & 31 & 11 & 5 & 3 \\
finish project & 65 & 20 & 8 & 6 \\
finish room & 79 & 25 & 16 & 10 \\
finish video & 44 & 16 & 9 & 6
\end{tabular}

more detailed information about meaning than would be given by a logical form that simply features enjoy $\left(e, x, e^{\prime}\right)$, where $e^{\prime}$ is the (event) variable that denotes the lecture.

Recall from Section 2.2 that thresholding is an option for the counts $f(v, e)$ and $f(o, e)$. We derived model paraphrases without employing any thresholds for these counts. Obtaining $f(v, e)$ from the parsed data was relatively straightforward, as there was no structural ambiguity involved. The parser's output was postprocessed to remove potentially erroneous information, so there was no reason to believe that the frequencies $f(v, e)$ and $f(o, e)$ were noisy. Furthermore, recent work has shown that omitting low-frequency tuples degrades performance for language-learning tasks such as PP attachment (Collins and Brooks 1995; Daelemans, van den Bosch, and Zavrel 1999), grapheme-to-phoneme conversion, POS tagging, and NP chunking (Daelemans, van den Bosch, and Zavrel 1999). For our task, employing thresholds for $f(v, e)$ and $f(o, e)$ dramatically decreases the number of derived interpretations. Table 8 shows the decrease in the number of interpretations as the cutoff for $f(v, e)$ and $f(o, e)$ is varied for five verb-object pairs that were included in our experimental study. Note that discarding counts occurring in the corpus only once reduces the number of interpretations by a factor of nearly three. Furthermore, applying frequency cutoffs reduces the range of the obtained probabilities: only likely (but not necessarily plausible) interpretations are obtained with $f(o, e) \geq 4$ and $f(v, e) \geq 4$. However, one of the aims of the experiment outlined below was to explore the quality of interpretations with varied probabilities. Table 9 displays the 10 most likely paraphrases (and their log-transformed probabilities) for finish room as the cutoff for the frequencies $f(v, e)$ and $f(o, e)$ is varied. Notice that applying a cutoff of three or four eliminates plausible interpretations such as decorate, wallpaper, furnish, and tidy. This may be particularly harmful for verb-noun (or adjective-noun) combinations that allow for a wide range of interpretations (like finish room).

We estimated the probability $P(e, o, v)$ for each verb-noun pair by varying the term $e$. In order to generate stimuli covering a wide range of paraphrases corresponding to different degrees of likelihood, for each verb-noun combination we divided the set of generated meanings into three "probability bands" (high, medium, and low) of equal size and randomly chose one interpretation from each band. This division ensured that subjects saw a wide range of paraphrases with different degrees of likelihood.

Our experimental design consisted of two factors: verb-noun pair (Pair) and probability band (Band). The factor Pair included 60 verb-noun combinations, and the factor Band had three levels, high, medium, and low. This yielded a total of Pair $\times$ Band $=60 \times 3=180$ stimuli. In order to limit the size of the experiment, the 180 stimuli were administered to two separate groups of subjects. The first group saw meaning paraphrases for the verbs attempt, begin, want, enjoy, try, and expect, whereas the sec- 
Table 9

Ten most likely interpretations for finish room (with log-transformed probabilities) as frequency threshold is varied.

\begin{tabular}{llllllll}
\hline \multicolumn{2}{c}{$f(v, e) \geq 1$} & \multicolumn{2}{c}{$f(v, e) \geq 2$} & \multicolumn{2}{c}{$f(v, e) \geq 3$} & \multicolumn{2}{c}{$f(v, e) \geq 4$} \\
\multicolumn{2}{c}{$f(o, e) \geq 1$} & & \multicolumn{2}{c}{$f(o, e) \geq 2$} & & $f(o, e) \geq 3$ & \multicolumn{2}{c}{$f(o, e) \geq 4$} \\
\hline decorate & -18.47 & decorate & -18.47 & fill & -18.88 & fill & -18.88 \\
wallpaper & -19.07 & fill & -18.89 & clean & -19.08 & clean & -19.08 \\
clean & -19.09 & clean & -19.08 & pack & -20.17 & pack & -20.17 \\
paper & -19.09 & search & -20.13 & make & -20.36 & make & -20.36 \\
furnish & -19.31 & pack & -20.17 & view & -20.78 & check & -21.24 \\
tidy & -19.92 & make & -20.36 & check & -21.24 & use & -21.78 \\
search & -20.13 & dress & -20.55 & pay & -21.53 & build & -21.96 \\
pack & -20.17 & view & -20.78 & use & -21.78 & give & -22.29 \\
make & -20.36 & check & -21.24 & build & -21.96 & prepare & -22.45 \\
view & -20.78 & paint & -21.38 & give & -22.29 & take & -23.11 \\
& & & & & & & \\
\hline
\end{tabular}

Table 10

Randomly selected example stimuli with log-transformed probabilities derived by the model.

\begin{tabular}{lllllll}
\hline Verb-noun & \multicolumn{2}{c}{ High } & \multicolumn{2}{c}{ Probability Band } \\
Medium & \multicolumn{2}{c}{ Low } \\
\hline attempt peak & climb & -20.22 & claim & -23.53 & include & -24.85 \\
begin production & organize & -19.09 & influence & -21.98 & tax & -22.79 \\
enjoy city & live in & -20.77 & come to & -23.50 & cut & -24.67 \\
expect reward & collect & -21.91 & claim & -23.13 & extend & -23.52 \\
finish room & wallpaper & -19.07 & construct & -22.49 & want & -24.60 \\
postpone payment & make & -21.85 & arrange & -23.21 & read & -25.92 \\
prefer people & talk to & -20.52 & sit with & -22.75 & discover & -25.26 \\
resist song & whistle & -22.12 & start & -24.47 & hold & -26.50 \\
start letter & write & -15.59 & study & -22.70 & hear & -24.50 \\
survive course & give & -22.88 & make & -24.48 & write & -26.27 \\
try drug & take & -17.81 & grow & -22.09 & hate & -23.88 \\
want hat & buy & -17.85 & examine & -21.56 & land on & -22.38
\end{tabular}

ond group saw paraphrases for finish, prefer, resist, start, postpone, and survive. Example stimuli are shown in Table 10.

Each experimental item consisted of two sentences, a sentence containing a metonymic construction (e.g., Peter started his dinner) and a sentence paraphrasing it (e.g., Peter started eating his dinner). The metonymic sentences and their paraphrases were created by the authors as follows. The selected verb-noun pairs were converted into simple sentences by adding a sentential subject and articles or pronouns where appropriate. The sentential subjects were familiar proper names (BNC corpus frequency $>$ 30 per million) balanced for gender. All sentences were in the past tense. In the paraphrasing sentences, the metonymy was spelled out by converting the model's output to a verb taking either a progressive or infinitive VP complement (e.g., started to eat or started eating). For verbs allowing both a progressive and an infinitive VP complement, we chose the type of complement with which the verb occurred more frequently in the corpus. A native speaker of English other than the authors was asked to confirm that the metonymic sentences and their paraphrases were syntactically well-formed (items found syntactically odd were modified and retested). Examples of the experi- 
mental stimuli the subjects saw are provided in (27) and (28). A complete list of the experimental items is given in Appendix B.

(27) a. high: Michael attempted the peak

Michael attempted to climb the peak

b. medium: Michael attempted the peak

Michael attempted to claim the peak

c. low: Michael attempted the peak

Michael attempted to include the peak

(28) a. high: Jean enjoyed the city

Jean enjoyed living in the city

b. medium: Jean enjoyed the city

Jean enjoyed coming to the city

c. low: Jean enjoyed the city

Jean enjoyed cutting the city

2.3.1.2 Procedure. The experimental paradigm was magnitude estimation (ME), a technique standardly used in psychophysics to measure judgments of sensory stimuli (Stevens 1975). The ME procedure requires subjects to estimate the magnitude of physical stimuli by assigning numerical values proportional to the stimulus magnitude they perceive. Highly reliable judgments can be achieved in this fashion for a wide range of sensory modalities, such as brightness, loudness, or tactile stimulation.

The ME paradigm has been extended successfully to the psychosocial domain (Lodge 1981), and recently Bard, Robertson, and Sorace (1996) and Cowart (1997) showed that linguistic judgments can be elicited in the same way as judgments of sensory or social stimuli. ME requires subjects to assign numbers to a series of linguistic stimuli in a proportional fashion. Subjects are first exposed to a modulus item, to which they assign an arbitrary number. All other stimuli are rated proportional to the modulus. In this way, each subject can establish his own rating scale, thus yielding maximally fine-grained data and avoiding the known problems with the conventional ordinal scales for linguistic data (Bard, Robertson, and Sorace 1996; Cowart 1997; Schütze 1996). In particular, ME does not restrict the range of the responses. No matter which modulus a subject chooses, he or she can subsequently assign a higher or lower judgment by using multiples or fractions of the modulus.

In the present experiment, each subject took part in an experimental session that lasted approximately 20 minutes. The experiment was self-paced, and response times were recorded to allow the data to be screened for anomalies. The experiment was conducted remotely over the Internet. Subjects accessed the experiment using their Web browser, which established an Internet connection to the experimental server running WebExp 2.1 (Keller, Corley, and Scheepers 2001), an interactive software package for administering Web-based psychological experiments. (For a discussion of WebExp and the validity of Web-based data, see Appendix A).

2.3.1.3 Instructions. Before participating in the actual experiment, subjects were presented with a set of instructions. The instructions explained the concept of numeric magnitude estimation of line length. Subjects were instructed to make estimates of line length relative to the first line they would see, the reference line. Subjects were told to give the reference line an arbitrary number, and then assign a number to each following line so that it represented how long the line was in proportion to the reference 
line. Several example lines and corresponding numerical estimates were provided to illustrate the concept of proportionality.

The subjects were instructed to judge how well a particular sentence paraphrased another sentence, using the same technique that they had applied to judging line length. Examples of plausible (see (29a)) and implausible (see (29b)) sentence paraphrases were provided, together with examples of numerical estimates.

(29) a. Peter started his dinner

b. Peter started his dinner

Peter started eating his dinner

Peter started writing his dinner

Subjects were informed that they would initially have to assign a number to a reference paraphrase. For each subsequent paraphrase, subjects were asked to assign a number indicating how good or bad that paraphrase was in proportion to the reference.

Subjects were told that they could use any range of positive numbers for their judgments, including decimals. It was stressed that there was no upper or lower limit on the numbers that could be used (exceptions being zero or negative numbers). Subjects were urged to use a wide range of numbers and to distinguish as many degrees of paraphrase plausibility as possible. It was also emphasized that there were no "correct" answers and that subjects should base their judgments on first impressions and not spend too much time thinking about any one paraphrase.

2.3.1.4 Demographic Questionnaire. After the instructions, a short demographic questionnaire was administered. The questionnaire asked subjects to provide their name, e-mail, address, age, sex, handedness, academic subject or occupation, and language region. Handedness was defined as "the hand you prefer to use for writing"; language region was defined as "the place (town, federal state, country) where you learned your first language."

2.3.1.5 Training Phase. The training phase was meant to familiarize subjects with the concept of numeric magnitude estimation using line lengths. Items were presented as horizontal lines, centered in the window of the subject's Web browser. After viewing an item, the subject had to provide a numerical judgment via the computer keyboard. After the subject pressed Return, the current item disappeared and the next item was displayed. There was no opportunity to revisit previous items or change responses once Return had been pressed. No time limit was set either for the item presentation or for the response, although response times were recorded for later inspection.

Subjects first judged the modulus item, and then all the items in the training set. The modulus was the same for all subjects, and it remained on the screen all the time to facilitate comparison. Items were presented in random order, with a new randomization being generated for each subject.

The training set contained six horizontal lines. The range of the shortest to longest item was one to ten (that is, the longest line was ten times the length of the shortest). The items were distributed evenly over this range, with the largest item covering the maximal window width of the Web browser. A modulus item in the middle of the range was provided.

2.3.1.6 Practice Phase. The practice phase enabled subjects to practice magnitude estimation of verb-noun paraphrases. Presentation and response procedure was the same as in the training phase, with linguistic stimuli being displayed instead of lines. Each subject judged the whole set of practice items, again presented to him or her in random order. 
The practice set consisted of eight paraphrase sentences that were representative of the test materials. The paraphrases were based on the three probability bands and represented a wide range of probabilities. A modulus item selected from the medium probability band was provided.

2.3.1.7 Experimental Phase. The presentation and response procedure in the experimental phase were the same as in the practice phase. Subjects were assigned to groups at random, and a random stimulus order was generated for each subject (for the complete list of experimental stimuli, see Appendix B). Each group of subjects saw 90 experimental stimuli (i.e., metonymic sentences and their paraphrases). As in the practice phase, the paraphrases were representative of the three probability bands (high, medium, low). Again a modulus item from the medium probability band was provided (see Appendix B). The modulus was the same for all subjects and remained on the screen the entire time the subject was completing the task.

2.3.1.8 Subject. Sixty-three native speakers of English participated in the experiment. The subjects were recruited over the Internet through advertisements posted to newsgroups and mailing lists. Participation was voluntary and unpaid. Subjects had to be linguistically naive (i.e., neither linguists nor students of linguistics were allowed to participate).

The data of two subjects were eliminated after inspection of their response times showed that they had not completed the experiment in a realistic time frame (i.e., they provided ratings too quickly, with average response time $<1000 \mathrm{~ms}$ ). The data of one subject were excluded because she was a non-native speaker of English.

This left 60 subjects for analysis. Of these, 53 subjects were right-handed, 7 lefthanded; 24 subjects were female, 36 male. The age of subjects ranged from 17 to 62; the mean was 26.4 years.

2.3.2 Results. The data were first normalized by dividing each numerical judgment by the modulus value that the subject had assigned to the reference sentence. This operation created a common scale for all subjects. Then the data were transformed by taking the decadic logarithm. This transformation ensured that the judgments were normally distributed and is standard practice for magnitude estimation data (Bard, Robertson, and Sorace 1996; Lodge 1981). All further analyses were conducted on the resulting normalized, log-transformed judgments.

We performed a correlation analysis to determine whether there was a linear relation between the paraphrases generated by the model and their perceived likelihood. This tested the hypothesis that meaning paraphrases assigned high probabilities by the model are perceived as better paraphrases by the subjects than meaning paraphrases assigned low probabilities. For each experimental item we computed the average of the normalized and log-transformed subject ratings. The mean subject ratings were then compared against the (log-transformed) probabilities assigned by the model for the same items.

The comparison between the absolute model probabilities and the human judgments yielded a Pearson correlation coefficient of .64 $(p<.01, N=174$; six items were discarded because of a coding error). The mean subject ratings and the model probabilities are given in Appendix B. Appendix C presents descriptive statistics for the model probabilities and the human judgments. The relationship between judgments and probabilities is plotted in Figure 1.

An important question is how well humans agree in their paraphrase judgments for verb-noun combinations. Intersubject agreement gives an upper bound for the 


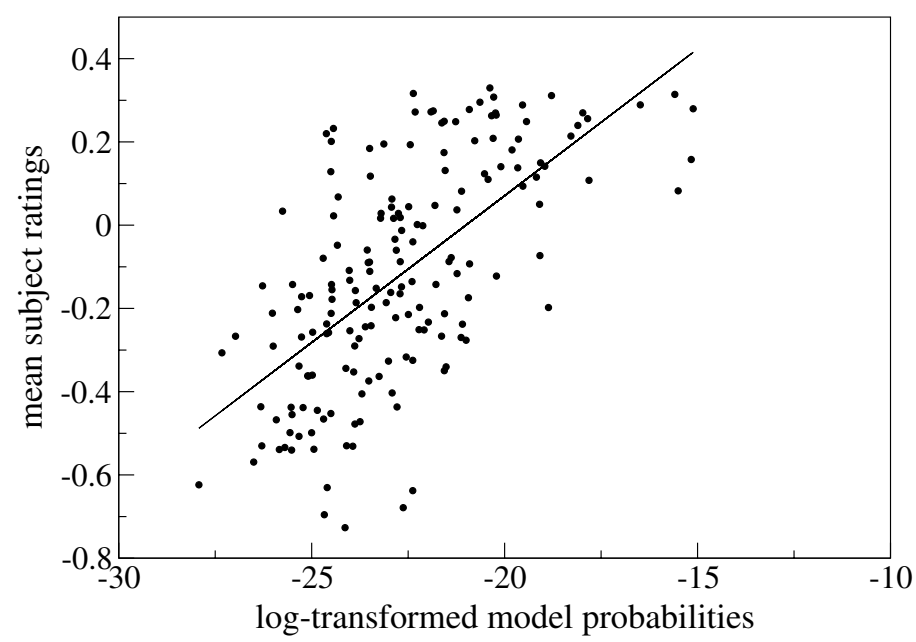

Figure 1

Correlation of elicited judgments and model-derived probabilities for metonymic verb-noun pairs.

task and allows us to interpret how well the model is doing in relation to humans. To calculate intersubject agreement, we used leave-one-out resampling. The technique is a special case of $n$-fold cross-validation (Weiss and Kulikowski 1991) and has been previously used for measuring how well humans agree on judging semantic similarity (Resnik and Diab 2000; Resnik 1999).

For each subject group we divided the set of the subjects' responses with size $m$ into a set of size $m-1$ (i.e., the response data of all but one subject) and a set of size one (i.e., the response data of a single subject). We then correlated the mean ratings of the former set with the ratings of the latter. This was repeated $m$ times. Since each group had 30 subjects, we performed 30 correlation analyses and report their mean. For the first group of subjects, the average intersubject agreement was .74 (Min $=.19$, Max $=.87$, StdDev $=.12$ ), and for the second group it was .73 (Min =.49, Max $=.87, \mathrm{StdDev}=.09)$. Our model's agreement with the human data is not far from the average human performance of .74 .

In the following section we introduce a naive model of verbal metonymy. We compare the naive model's performance against the human judgments and the paraphrases generated by our initial model. We discuss extensions of the basic model in Section 2.5.1.

\subsection{Experiment 2: Comparison against Naive Baseline}

2.4.1 Naive Baseline Model. In the case of verbal metonymy a naive baseline model can be constructed by simply taking verb-noun co-occurrence data into account, ignoring thus the dependencies between the polysemous verb and its progressive or infinitival VP complements. Consider the sentence John began the book. In order to generate appropriate paraphrases for begin book, we will consider solely the verbs that take book as their object (i.e., read, write, buy, etc.). This can be simply expressed as $P(e \mid o)$, the conditional probability of a verb $e$ given its object $o$ (i.e., the noun figuring in the metonymic expression), which we estimate as follows:

$$
\hat{P}(e \mid o)=\frac{f(e, o)}{f(o)}
$$


The model in (30) treats metonymic verbs as semantically empty and relies on their object NPs to provide additional semantic information. The counts $f(e, o)$ and $f(o)$ can be easily obtained from the BNC: $f(o)$ amounts to the number of times a noun is attested as an object, and $f(e, o)$ are verb-object tuples extracted from the BNC using Cass (Abney 1996) as described earlier.

2.4.2 Results. We used the naive model to calculate the likelihood of the meaning paraphrases that were presented to the subjects (see Experiment 1). Through correlation analysis we explored the linear relationship between the elicited judgments and the naive baseline model. We further directly compared the two models: that is, our initial, linguistically more informed model and the naive baseline.

Comparison between the probabilities generated by the naive model and the elicited judgments yielded a Pearson correlation coefficient of .42 $(p<.01, N=174)$. (Recall that our initial model yielded a correlation coefficient of .64.) We conducted a one-tailed $t$-test to determine if the correlation coefficients were significantly different. The comparison revealed that the difference between them was statistically significant $(t(171)=1.67, p<.05)$, indicating that our model performs reliably better than the naive baseline. Comparison between the two models (our initial model introduced in Section 2.1 and the naive baseline model) yielded an intercorrelation of $.46(p<.01$, $N=174$ ). These differences between the "full" probabilistic model and the naive baseline model confirm claims made in the literature: Different metonymic verbs have a different semantic impact on the resolution of metonymy.

\subsection{Experiment 3: Comparison against Norming Data}

Our previous experiments focused on evaluating the plausibility of meaning paraphrases generated by a model that does not take into account the contribution of the sentential subject. However, properties of the subject NP appear to influence the interpretation of the metonymic expression in otherwise neutral contexts, as is illustrated in (31), in which the interpretation of enjoy the book is influenced by the sentential subject: Authors usually write books, whereas critics usually review them.

(31) a. The critic enjoyed the book.

b. The author enjoyed the book.

In this section we present an extension of the basic model outlined in Section 2.1 that takes sentential subjects into account. We evaluate the derived paraphrases and their likelihood again by comparison with human data. This time we compare our model against paraphrase data generated independently by subjects that participated in an experimental study (McElree et al. 2001) that was not designed specifically to test our model.

2.5.1 The Extended Model. We model the meaning of sentences like (31) again as the joint distribution of the following variables: the metonymic verb $v$, its subject $s$, its object $o$, and the implicit interpretation $e$. By choosing the ordering $\langle e, v, s, o\rangle$, we can factor $P(e, o, s, v)$ as follows:

$$
P(e, o, s, v)=P(e) \cdot P(v \mid e) \cdot P(s \mid e, v) \cdot P(o \mid e, v, s)
$$

The terms $P(e)$ and $P(v \mid e)$ are easy to estimate from the BNC. For $P(e)$ all we need is a POS-tagged corpus and $P(v \mid e)$ can be estimated from Cass's output (see equations (12) and (13)). The estimation of the terms $P(s \mid e, v)$ and $P(o \mid e, v, s)$ is, however, 
problematic, as they rely on the frequencies $f(s, e, v)$ and $f(o, e, v, s)$, respectively. Recall that there is a discrepancy between a metonymic interpretation and its usage. As we discussed earlier, metonymic interpretation is not overtly expressed in the corpus. Furthermore, the only type of data available to us for the estimation of $P(s \mid e, v)$ and $P(o \mid e, v, s)$ is the partially parsed BNC, which is not annotated with information regarding the interpretation of metonymies. This means that $P(s \mid e, v)$ and $P(o \mid e, v, s)$ need to be approximated somehow. We first assume that the sentential subject $s$ is conditionally independent of the metonymic verb $v$; second, we assume that the sentential object $o$ is conditionally independent of $v$ and $s$ :

$$
\begin{gathered}
P(s \mid e, v) \approx P(s \mid e) \\
P(o \mid e, v, s) \approx P(o \mid e)
\end{gathered}
$$

The rationale behind the approximation in (33) is that the likelihood of a noun $s$ being a subject of a verb $e$ is largely independent of whether $e$ is the complement of a metonymic verb $v$. For example, authors usually write, irrespective of whether they enjoy, dislike, start, or finish doing it. The motivation for the approximation in (34) comes from the observation that an object is more closely related to the verb that selects for it than a subject or a metonymic verb. We are likely to come up with book or letter for $o$ if we know that $o$ is the object of read or write. Coming up with an object for $o$ is not so straightforward if all we know is the metonymic verb (e.g., enjoy, finish) or its sentential subject. It is the verbs, rather than other sentential constituents, that impose semantic restrictions on their arguments. We estimate $P(s \mid e)$ and $P(o \mid e)$ using maximum likelihood:

$$
\begin{aligned}
& \hat{P}(s \mid e)=\frac{f(s, e)}{f(e)} \\
& \hat{P}(o \mid e)=\frac{f(o, e)}{f(e)}
\end{aligned}
$$

The count $f(s, e)$ amounts to the number of times a noun $s$ is attested as the subject of a verb $e ; f(o, e)$ represents the number of times a noun is attested as an object of $e$. Verb-argument tuples can be easily extracted from the BNC using Cass (see Section 2.2 for details). Table 11 illustrates the model's performance for the metonymic constructions in (37). The table shows only the five most likely interpretations the model came up with for each construction. Interestingly, different interpretations are derived for different subjects. Even though pianists and composers are semantically related, pianists are more likely to begin playing a symphony, whereas composers are more likely to conduct or write a symphony. Similarly, builders tend to renovate houses and architects tend to design them.

(37) a. The composer/pianist began the symphony.

b. The author/student started the book.

c. The builder/architect started the house.

d. The secretary/boss finished the memo.

In the following section we compare the interpretations generated by the model against paraphrases provided by humans. More specifically, we explore whether there is a linear relationship between the frequency of an interpretation as determined in a norming study and the probability of the same interpretation as calculated by the model. 
Table 11

Subject-related model interpretations, ranked in order of likelihood.

\begin{tabular}{|c|c|c|c|c|c|c|c|}
\hline \multicolumn{4}{|c|}{ begin symphony } & \multicolumn{4}{|c|}{ start book } \\
\hline \multicolumn{2}{|c|}{ composer } & \multicolumn{2}{|c|}{ pianist } & \multicolumn{2}{|c|}{ author } & \multicolumn{2}{|c|}{ student } \\
\hline $\begin{array}{l}\text { write } \\
\text { conduct } \\
\text { hear } \\
\text { play } \\
\text { create }\end{array}$ & $\begin{array}{l}-22.2 \\
-23.79 \\
-25.38 \\
-25.81 \\
-25.96\end{array}$ & $\begin{array}{l}\text { play } \\
\text { hear } \\
\text { give } \\
\text { do } \\
\text { have }\end{array}$ & $\begin{array}{l}-24.20 \\
-25.38 \\
-28.44 \\
-29.23 \\
-30.13\end{array}$ & $\begin{array}{l}\text { write } \\
\text { publish } \\
\text { compile } \\
\text { read } \\
\text { sign }\end{array}$ & $\begin{array}{l}-14.87 \\
-16.94 \\
-17.84 \\
-17.98 \\
-18.59\end{array}$ & $\begin{array}{l}\text { read } \\
\text { write } \\
\text { study } \\
\text { research } \\
\text { translate }\end{array}$ & $\begin{array}{l}-16.12 \\
-16.48 \\
-17.59 \\
-18.86 \\
-17.85\end{array}$ \\
\hline \multicolumn{4}{|c|}{ start house } & \multicolumn{4}{|c|}{ finish memo } \\
\hline \multicolumn{2}{|c|}{ builder } & \multicolumn{2}{|c|}{ architect } & \multicolumn{2}{|c|}{ secretary } & \multicolumn{2}{|c|}{ boss } \\
\hline $\begin{array}{l}\text { renovate } \\
\text { build } \\
\text { demolish } \\
\text { dismantle } \\
\text { erect }\end{array}$ & $\begin{array}{l}-15.43 \\
-17.56 \\
-18.37 \\
-19.69 \\
-19.81\end{array}$ & $\begin{array}{l}\text { design } \\
\text { build } \\
\text { restore } \\
\text { purchase } \\
\text { site }\end{array}$ & $\begin{array}{l}-16.87 \\
-17.20 \\
-19.08 \\
-19.32 \\
-19.73\end{array}$ & $\begin{array}{l}\text { write } \\
\text { type } \\
\text { send } \\
\text { sign } \\
\text { make }\end{array}$ & $\begin{array}{l}-19.79 \\
-20.13 \\
-20.87 \\
-22.01 \\
-22.74\end{array}$ & $\begin{array}{l}\text { draft } \\
\text { send } \\
\text { sign } \\
\text { hand } \\
\text { write }\end{array}$ & $\begin{array}{l}-20.73 \\
-21.97 \\
-22.04 \\
-22.12 \\
-22.74\end{array}$ \\
\hline
\end{tabular}

2.5.2 Method. For the experiment described in this section, we used the norming data reported in McElree et al. (2001). In McElree et al.'s study subjects were given sentence fragments such as (38) and were asked to complete them. Potential completions for fragment (38a) include writing or reading. The study consisted of 142 different sentences similar to those shown in (38) and included 15 metonymic verbs. Thirty sentences were constructed for each of the metonymic verbs start, begin, complete, and finish, and a total of 22 sentences for attempt, endure, expect, enjoy, fear, master, prefer, resist, savor, survive, and try.

(38) a. The writer finished _ the novel.

b. The soldier attempted _ the mountain.

c. The teenager finished _ the novel.

The completions can be used to determine interpretation preferences for the metonymic constructions simply by counting the verbs that human subjects use to complete sentences like those in (38). For example, five completions were provided by the subjects for fragment (38b): climb, hike, scale, walk, and take. Of these climb was by far the most likely, with 78 (out of 88) subjects generating this interpretation. ${ }^{3}$ The most likely interpretations for (38a) and (38c) were, respectively, write (13 out of 28 subjects) and read (18 out of 22$)$.

For each of the sentences included in McElree et al.'s (2001) study, we derived interpretation paraphrases using the model presented in Section 2.5.1. We next compared the interpretations common in the model and the human data.

2.5.3 Results. In Experiment 1 we evaluated the paraphrases generated by the model by eliciting plausibility judgments from subjects and showed that our model produces an intuitively plausible ranking of meanings. Here, we evaluate the quality of the

3 McElree et al.'s (2001) linguistic materials were manually constructed and not controlled for frequency. For example, one would expect (38b) to be relatively rare, even in a large corpus. This is true for the $\mathrm{BNC}$, in which the combination attempt mountain is not attested at all. 
produced paraphrases by directly comparing them to norming data acquired independently of our model and the particular corpus we are using.

The comparison between (log-transformed) model probabilities and (log-transformed) completion frequencies yielded a Pearson correlation coefficient of .422 $(p<.01$, $N=341$ ). We also compared the completion frequencies against interpretation probabilities derived using the model presented in Section 2.3, which does not take subjectrelated information into account. The comparison yielded a correlation coefficient of $.216(p<.01, N=341)$. We carried out a one-tailed $t$-test to determine if the difference between the two correlation coefficients is significant. The comparison revealed that the difference is statistically significant $(t(338)=2.18, p<.05)$. This means that the fit between the norming data and the model is better when the model explicitly incorporates information about the sentential subject. The two models are, as expected, intercorrelated $(r=.264, p<.01, N=341)$.

\subsection{Discussion}

We have demonstrated that the meanings acquired by our probabilistic model correlate reliably with human intuitions. These meanings go beyond the examples found in the theoretical linguistics literature. The verb-noun combinations we interpret were randomly sampled from a large, balanced corpus providing a rich inventory for their meanings. We have shown that the model has four defining features: (1) It is able to derive intuitive meanings for verb-noun combinations, (2) it generates clusters of meanings (following Vendler's (1968) insight), (3) it predicts variation in interpretation among the different nouns: The same verb may carry different meanings depending on its subject or object (compare begin book to begin house and the author began the house to the architect began the house), and (4) it represents variation in interpretation among the different metonymic verbs (e.g., begin book vs. enjoy book). This latter property demonstrates that although the model does not explicitly encode linguistic constraints for resolving metonymies, it generates interpretations that broadly capture linguistic differences (e.g., attempt imposes different constraints on interpretation from begin or enjoy). Furthermore, these interpretations for metonymic verb-noun pairs are discovered automatically, without presupposing the existence of a predefined taxonomy or a knowledge base.

Note that the evaluation procedure to which we subject our model is rather strict. The derived verb-noun combinations were evaluated by subjects naive to linguistic theory. Although verbal logical metonymy is a well-researched phenomenon in the theoretical linguistics literature, the experimental approach advocated here is, to our knowledge, new. Comparison between our model and human judgments yielded a reliable correlation of .64 when the upper bound for the task (i.e., intersubject agreement) is on average .74. Furthermore, our model performed reliably better than a naive baseline model, which achieved a correlation of only .42. When compared against norming data, an extended version of our model that takes subject information into account reached a correlation of .42. Comparison against norming data is a strict test on unseen data that was not constructed explicitly to evaluate our model but is independently motivated and does not take our corpus (i.e., the BNC) or our particular task into account.

We next investigate whether such an approach generalizes to other instances of logical metonymy by looking at adjective-noun combinations. Adjectives pose a greater challenge for our modeling task, as they can potentially allow for a wider range of interpretations and can exhibit preferences for a verb-subject or verb-object paraphrase (see Section 1). Following the approach we adopted for verbal metonymy, we define the interpretation of polysemous adjective-noun combinations as a paraphrase gener- 
ation task. We provide a probabilistic model that not only paraphrases adjective-noun pairs (e.g., fast plane) with a related verb (e.g., fly) but also predicts whether the noun modified by the adjective (e.g., plane) is likely to be the verbal object or subject. The model achieves this by combining distributional information about how likely it is for any verb to be modified by the adjective in the adjective-noun combination or its corresponding adverb with information about how likely it is for any verb to take the modified noun as its object or subject. We obtain quantitative information about verbadjective modification and verb-argument relations from the BNC and evaluate our results by comparing the model's predictions against human judgments. Consistent with our results on verbal metonymy, we show that the model's ranking of meanings correlates reliably with human intuitions.

\section{Metonymic Adjectives}

\subsection{The Model}

Consider again the adjective-noun combinations in (39). In order to come up with the interpretation of plane that flies quickly for fast plane, we would like to find in the corpus a sentence whose subject is the noun plane or planes and whose main verb is fly. We would also expect fly to be modified by the adverb fast or quickly. In the general case, we would like to gather from the corpus sentences indicating what planes do fast. Similarly, for the adjective-noun combination fast scientist, we would like to find in the corpus information indicating what the activities that scientists perform fast are, whereas for easy problem we need information about what one can do with problems easily (e.g., one can solve problems easily) or about what problems are (e.g., easy to solve or easy to set).
(39) a. fast plane
b. fast scientist
c. fast programmer
d. easy problem

In sum, in order to come up with a paraphrase of the meaning of an adjectivenoun combination, we need to know which verbs take the head noun as their subject or object and are modified by an adverb corresponding to the modifying adjective. This can be expressed as the joint probability $P(a, e, n, r e l)$, where $e$ is the verbal predicate modified by the adverb $a$ (directly derived from the adjective present in the adjectivenoun combination) bearing the argument relation rel (i.e., subject or object) to the head noun $n$. By choosing the ordering $\langle e, n, a, r e l\rangle$ for the variables $a, e, n$, and $r e l$, we can rewrite $P(a, e, n, r e l)$, using the chain rule, as follows:

$$
P(a, e, n, r e l)=P(e) \cdot P(n \mid e) \cdot P(a \mid e, n) \cdot P(r e l \mid e, n, a)
$$

Although the terms $P(e)$ and $P(n \mid e)$ can be straightforwardly estimated from the BNC (see (12) for $P(e) ; P(n \mid e)$ can be obtained by counting the number of times a noun $n$ co-occurs with a verb $e$ either as its subject or object), the estimation of $P(a \mid e, n)$ and $P(r e l \mid e, n, a)$ faces problems similar to those for metonymic verbs. Let us consider more closely the term $P(r e l \mid e, n, a)$, which can be estimated as shown in (41).

$$
\hat{P}(r e l \mid e, n, a)=\frac{f(r e l, e, n, a)}{f(e, n, a)}
$$


One way to obtain $f(r e l, e, n, a)$ would be to parse fully the corpus so as to identify the verbs that take the head noun $n$ as their subject or object and are modified by the adverb $a$, assuming it is equally likely to find in a corpus the metonymic expression (e.g., fast plane) and its paraphrase interpretation (i.e., plane that flies quickly). As in the case of verb-noun metonymies, this assumption is unjustified: For the adjective-noun combination fast plane, there are only six sentences in the entire BNC that correspond to $f(r e l, e, n, a)$. According to the sentences in (42), the most likely interpretation for fast plane is plane that goes fast (see examples (42a)-(42c)). The interpretations plane that swoops in fast, plane that drops down fast, and plane that flies fast are all equally likely, since they are attested in the corpus only once (see examples (42d)-(42f)). This is rather unintuitive, since fast planes are more likely to fly than swoop in fast. Similar problems affect the frequency $f(e, n, a)$.

(42) a. The plane went so fast it left its sound behind.

b. And the plane's going slightly faster than the Hercules or Andover.

c. He is driven by his ambition to build a plane that goes faster than the speed of sound.

d. Three planes swooped in, fast and low.

e. The plane was dropping down fast towards Bangkok.

f. The unarmed plane flew very fast and very high.

In default of a corpus explicitly annotated with interpretations for metonymic adjectives, we will make the following independence assumptions:

$$
\begin{gathered}
P(a \mid e, n) \approx P(a \mid e) \\
P(r e l \mid e, n, a) \approx P(r e l \mid e, n)
\end{gathered}
$$

The rationale behind the approximation in (43) is that the likelihood of seeing an adverbial $a$ modifying a verb $e$ bearing an argument relation to a noun $n$ is largely independent of that specific noun. For example, flying can be carried out fast or slowly or beautifully irrespective of whether it is a pilot or a bird who is doing the flying. Similarly, the adverb peacefully is more related to dying than killing or injuring irrespective of who the agent of these actions is. Accordingly, we assume that the argument relation rel is independent of whether the verb $e$ (standing in relation rel with noun $n$ ) is modified by an adverb $a$ (see (44)). In other words, it is the verb $e$ and its argument $n$ that determine the relation rel rather than the adjective or adverb $a$. Knowing that flying is conducted slowly will not affect the likelihood of inferring a subject relation for plane and fly. Yet we are likely to infer an object relation for plane and construct irrespective of whether the constructing is done slowly, quickly, or automatically. We estimate the probabilities $P(e), P(n \mid e), P(a \mid e)$, and $P(r e l \mid e, n)$ as follows:

$$
\begin{aligned}
\hat{P}(e) & =\frac{f(e)}{N} \\
\hat{P}(n \mid e) & =\frac{f(n, e)}{f(e)} \\
\hat{P}(a \mid e) & =\frac{f(a, e)}{f(e)}
\end{aligned}
$$


Table 12

Most frequent verbs modified by the adverb fast.

\begin{tabular}{lrll}
\hline & \multicolumn{2}{c}{$f($ fast,$e)$} & \\
\hline go & 29 & work,$e)$ & 6 \\
grow & 28 & grow in & 6 \\
beat & 27 & learn & 5 \\
run & 16 & happen & 5 \\
rise & 14 & walk & 4 \\
travel & 13 & think & 4 \\
move & 12 & keep up & 4 \\
come & 11 & fly & 4 \\
drive & 8 & fall & 4 \\
get & 7 & disappear & 4 \\
& & & \\
\hline
\end{tabular}

Table 13

Most frequent verbs taking as an argument the noun plane.

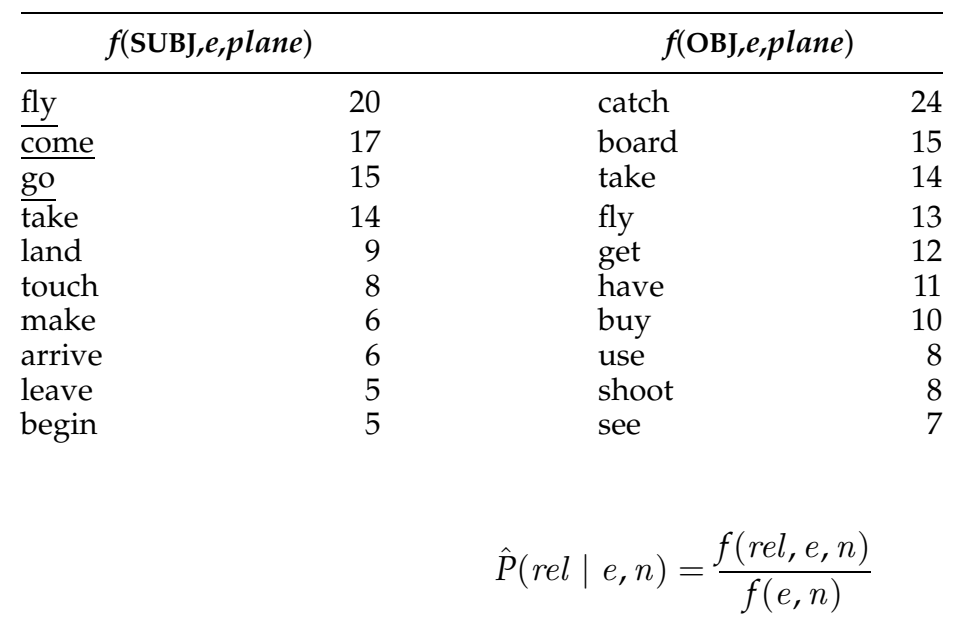

By substituting equations (45)-(48) into (41) and simplifying the relevant terms, (41) can be rewritten as follows:

$$
P(a, e, n, r e l)=\frac{f(r e l, e, n) \cdot f(a, e)}{f(e) \cdot N}
$$

Assume we want to discover a meaning paraphrase for the adjective-noun combination fast plane. We need to find the verb $e$ and the relation rel (i.e., subject or object) that maximize the term $P($ fast, $e$, plane, $r e l)$. Table 12 gives a list of the most frequent verbs modified by the adverb fast in the BNC (see the term $f(a, e)$ in equation (49)), and Table 13 lists the verbs for which the noun plane is the most likely object or subject (see the term $f(r e l, e, n)$ in equation (49)). In the following section, we describe how the frequencies $f(r e l, e, n), f(a, e)$, and $f(e)$ were estimated from a lemmatized version of the BNC.

Table 12 can be thought of as a list of the activities that can be fast (i.e., going, growing, flying), whereas Table 13 specifies the events associated with the noun plane. Despite our simplifying assumptions, the model given in (49) will come up with plausible meanings for adjective-noun combinations like fast plane. Note that the verbs fly, come, and go are most likely to take the noun plane as their subject (see Table 13). These 
verbs also denote activities that are fast (see Table 12, in which the underlined verbs are events that are associated both with the adverb fast and the noun plane). Further note that a subject interpretation is more likely than an object interpretation for fast plane, since none of the verbs likely to have plane as their object are modified by the adverb fast (compare Tables 12 and 13).

As in the case of metonymic verbs, the probabilistic model outlined above acquires meanings for polysemous adjective-noun combinations in an unsupervised manner without presupposing annotated corpora or taxonomic information. The obtained meanings are not discourse-sensitive; they can be thought of as default semantic information associated with a particular adjective-noun combination. This means that our model is unable to predict that programmer that runs fast is a likely interpretation for fast programmer when the latter is in a context like the one given in (5) (repeated here as (50)).

(50) a. All the office personnel took part in the company sports day last week.

b. One of the programmers was a good athlete, but the other was struggling to finish the courses.

c. The fast programmer came first in the $100 \mathrm{~m}$.

\subsection{Parameter Estimation}

As in the case of verbs, the parameters of the model were estimated using a part-ofspeech-tagged and lemmatized version of the BNC. The counts $f(e)$ and $N$ (see (49)) reduce to the number of times a given verb is attested in the corpus. The frequency $f(r e l, e, n)$ was obtained using Abney's (1996) chunk parser Cass (see Section 2.2 for details).

Generally speaking, the frequency $f(a, e)$ represents not only a verb modified by an adverb derived from the adjective in question (see example (51a)), but also constructions like the ones shown in (51b) and (51c), in which the adjective takes an infinitival VP complement whose logical subject can be realized as a for PP (see example (51c)). In cases of verb-adverb modification we assume access to morphological information that specifies what counts as a valid adverb for a given adjective. In most cases adverbs are formed by adding the suffix -ly to the base of the adjective (e.g., slow-ly, easy-ly). Some adjectives have identical adverbs (e.g., fast, right). Others have idiosyncratic adverbs (e.g., the adverb of good is well). It is relatively straightforward to develop an automatic process that maps an adjective to its corresponding adverb, modulo exceptions and idiosyncracies; however, in the experiments described in the following sections, this mapping was manually specified.

(51) a. comfortable chair $\rightarrow$ a chair on which one sits comfortably

b. comfortable chair $\rightarrow$ a chair that is comfortable to sit on

c. comfortable chair $\rightarrow$ a chair that is comfortable for me to sit on

In cases in which the adverb does not immediately succeed the verb, the parser is not guaranteed to produce a correct analysis, since it does not resolve structural ambiguities. So we adopted a conservative strategy, in which to obtain the frequency $f(a, e)$, we looked only at instances in which the verb and the adverbial phrase modifying it were adjacent. More specifically, in cases in which the parser identified an AdvP following a VP, we extracted the verb and the head of the AdvP (see the examples in (52)). In cases where the AdvP was not explicitly identified, we extracted the verb and the adverb immediately following or preceding it (see the examples in (53)), 
assuming that the verb and the adverb stand in a modification relation. The examples below illustrate the parser's output and the information that was extracted for the frequency $f(a, e)$.

(52) a. [NP Oriental art] [vP came] [AdvP more slowly.] come slowly

b. [NP The issues] [vP will not be resolved] [AdvP easily.] resolve easily

c. [NP Arsenal] [vp had been pushed] [AdvP too hard.] push hard

(53) a. [NP Some art historians] [vP write well] [PP about the present.] write well

b. [NP The accidents] [vP could have been easily avoided.] avoid easily

c. [NP A system of molecules] [vP is easily shown] [vP to stay constant.] show easily

d. [NP Their economy] [vp was so well run.] run well

Adjectives with infinitival complements (see (51b) and (51c)) were extracted from the parser's output. We concentrated solely on adjectives immediately followed by infinitival complements with an optionally intervening for PP (see (51c)). The adjective and the main verb of the infinitival complement were counted as instances of the quantity $f(a, e)$. The examples in (54) illustrate the process.

(54) a. [NP These early experiments] [VP were easy] [vP to interpret.] easy interpret

b. [NP It] [VP is easy] [PP for an artist] [vP to show work independently.] easy show

c. [NP It] [vp is easy] [vp to show] [vp how the components interact.] easy show

Finally, the frequency $f(a, e)$ collapsed the counts from cases in which the adjective was followed by an infinitival complement (see the examples in (54)) and cases in which the verb was modified by the adverb corresponding to the related adjective (see the examples in (52)-(53)). For example, assume that we are interested in the frequency $f$ (easy, show). In this case, we will take into account not only sentences (54b) and (54c), but also sentence (53b). Assuming this was the only evidence in the corpus, the frequency $f$ (easy, show) would be three.

Once we have obtained the frequencies $f(a, e)$ and $f(r e l, e, n)$, we can determine what the most likely interpretations for a given adjective-noun combination are. If we know the interpretation preference of a given adjective (i.e., subject or object), we may vary only the term $e$ in $P(a, n, r e l, e)$, keeping the terms $n, a$, and rel constant. Alternatively, we could acquire the interpretation preferences automatically by varying both the terms rel and $e$. In Experiment 4 (see Section 3.3) we acquire both meaning paraphrases and argument preferences for polysemous adjective-noun combinations.

In what follows we illustrate the properties of the model by applying it to a small number of adjective-noun combinations (displayed in Table 14). The adjective-noun 
Table 14

Paraphrases for adjective-noun combinations taken from the literature.

\begin{tabular}{llr}
\hline easy problem & $\rightarrow$ a problem that is easy to solve & (Vendler 1968, page 97) \\
easy text & $\rightarrow$ text that reads easily & (Vendler 1968, page 99) \\
difficult language & $\rightarrow$ a language that is difficult to speak, learn, write, understand & (Vendler 1968, page 99) \\
careful scientist & $\rightarrow$ a scientist who observes, performs, runs experiments carefully & (Vendler 1968, page 92) \\
$\begin{array}{l}\text { comfortable chair } \\
\text { good umbrella }\end{array}$ & $\rightarrow$ a chair on which one sits comfortably & (Vendler 1968, page 98) \\
& $\rightarrow$ an umbrella that functions well & (Pustejovsky 1995, page 43) \\
\hline
\end{tabular}

Table 15

Object-related interpretations for adjective-noun combinations, ranked in order of likelihood.

\begin{tabular}{lllllllllll}
\hline \multicolumn{2}{c}{ easy problem } & \multicolumn{2}{c}{ easy text } & \multicolumn{2}{c}{ difficult language } & \multicolumn{2}{c}{ comfortable chair } & good umbrella \\
\hline solve & -15.14 & read & -17.42 & understand & -17.15 & sink into & -18.66 & keep & -21.59 \\
deal with & -16.12 & handle & -18.79 & interpret & -17.59 & sit on & -19.13 & wave & -21.61 \\
identify & -16.83 & use & -18.83 & learn & -17.67 & lounge in & -19.15 & hold & -21.73 \\
tackle & -16.92 & interpret & -19.05 & use & -17.79 & relax in & -19.33 & run for & -21.73 \\
handle & -16.97 & understand & -19.15 & speak & -18.21 & nestle in & -20.51 & leave & -22.28 \\
& & & & & & & & & & \\
\end{tabular}

Table 16

Subject-related interpretations for adjective-noun combinations, ranked in order of likelihood.

\begin{tabular}{|c|c|c|c|c|c|}
\hline \multicolumn{2}{|c|}{ easy text } & \multicolumn{2}{|c|}{ good umbrella } & \multicolumn{2}{|c|}{ careful scientist } \\
\hline $\begin{array}{l}\text { see } \\
\text { read } \\
\text { understand } \\
\text { achieve } \\
\text { explain }\end{array}$ & $\begin{array}{l}-19.22 \\
-19.50 \\
-19.66 \\
-19.71 \\
-20.40\end{array}$ & cover & -23.05 & $\begin{array}{l}\text { calculate } \\
\text { proceed } \\
\text { investigate } \\
\text { study } \\
\text { analyze }\end{array}$ & $\begin{array}{l}-22.31 \\
-22.67 \\
-22.78 \\
-22.90 \\
-22.92\end{array}$ \\
\hline
\end{tabular}

combinations and their respective interpretations are taken from the lexical semantics literature (i.e., Pustejovsky (1995) and Vendler (1968)). The five most likely modelderived paraphrases for these combinations are shown in Tables 15 and 16.

The model comes up with plausible meanings, some of which overlap with those suggested in the lexical semantics literature (underlined interpretations indicate agreement between the model and the literature). Observe that the model predicts different meanings when the same adjective modifies different nouns and derives a cluster of meanings for a single adjective-noun combination. An easy problem is not only a problem that is easy to solve (see Vendler's (1968) identical interpretation in Table 14) but also a problem that is easy to deal with, identify, tackle, and handle. The meaning of easy problem is different from the meaning of easy text, which in turn is easy to read, handle, interpret, and understand. The interpretations the model arrives at for difficult language are a superset of the interpretations suggested by Vendler (1968). The model comes up with the additional meanings language that is difficult to interpret and language that is difficult to use. Although the meanings acquired by the model for careful scientist do not overlap with the ones suggested by Vendler (1968), they seem intuitively plausible: a careful scientist is a scientist who calculates, proceeds, investigates, studies, and analyzes carefully. These are all possible actions associated with scientists.

The model derives subject- and object-related interpretations for good umbrella, which is an umbrella that covers well and is good to keep, good for waving, good to hold, good to run for, and good to leave. A subject interpretation can be also derived for 
easy text. Our parser treats inchoative and noninchoative uses of the same verb as distinct surface structures (e.g., text that one reads easily vs. text that reads easily); as a result, read is generated as a subject and object paraphrase for easy text (compare Tables 15 and 16). The object-related interpretation is nevertheless given a higher probability than the subject-related one, which seems intuitively correct (there is an understood but unexpressed agent in structures like text that reads easily). In general, subject and object interpretations are derived on the basis of verb-subject and verb-object constructions that have been extracted from the corpus heuristically without taking into account information about theta roles, syntactic transformations (with the exception of passivization), or diathesis alternations such as the middle or causative/inchoative alternation.

Although the model can be used to provide several interpretations for a given adjective-noun combination, not all of these interpretations are useful or plausible (see the subject interpretations for easy text). Also, the meanings acquired by our model are a simplified version of the ones provided in the lexical semantics literature. An adjective-noun combination may be paraphrased with another adjective-noun combination (e.g., a good meal is a tasty meal) or with an NP instead of an adverb (e.g., a fast decision is a decision that takes a short amount of time). We are making the simplifying assumption that a polysemous adjective-noun combination can be paraphrased by a sentence consisting of a verb whose argument is the noun with which the adjective is in construction (cf. earlier discussion concerning nonmetonymic uses of verbs like enjoy).

In the following section we evaluate against human judgments the meaning paraphrases generated by the model. As in the case of verbs, the model is tested on examples randomly sampled from the $\mathrm{BNC}$, and the linear relationship between the subjects' rankings and the model-derived probabilities is explored using correlation analysis. In Section 3.5.1 we assess whether our model outperforms a naive baseline in deriving interpretations for metonymic adjectives.

\subsection{Experiment 4: Comparison against Human Judgments}

The experimental method in Experiment 4 was the same as that in Experiment 1. Meaning paraphrases for adjective-noun combinations were obtained using the model introduced in Section 3.1. The model's rankings were compared against paraphrase judgments elicited experimentally from human subjects. The comparison between model probabilities and their perceived likelihood enabled us to explore (1) whether there is a linear relationship between the likelihood of a given meaning as derived by the model and its perceived plausibility and (2) whether the model can be used to derive the argument preferences for a given adjective, that is, whether the adjective is biased toward a subject or object interpretation or whether it is equibiased.

\subsubsection{Method.}

3.3.1.1 Materials and Design. We chose nine adjectives according to a set of minimal criteria and paired each adjective with 10 nouns randomly selected from the BNC. We chose the adjectives as follows: We first compiled a list of all the polysemous adjectives mentioned in the lexical semantics literature (Vendler 1968; Pustejovsky 1995). From these we randomly sampled nine adjectives (difficult, easy, fast, good, hard, right, safe, slow, and wrong). These adjectives had to be relatively unambiguous syntactically: In fact, these nine adjectives were unambiguously tagged as "adjectives" $98.6 \%$ of the time, measured as the number of different part-of-speech tags assigned to the word in the BNC. The nine selected adjectives ranged in BNC frequency from 57.6 per million to 1,245 per million. 
Adjective-noun pairs were extracted from the parser's output. Recall that the BNC was parsed using Abney's (1996) chunker Cass (see Sections 2.2 and 3.2 for details). From the syntactic analysis provided by the parser, we extracted a table containing the adjective and the head of the noun phrase following it. In the case of compound nouns, we included only sequences of two nouns and considered the rightmost-occurring noun as the head. From the retrieved adjective-noun pairs, we removed all pairs with BNC frequency of one, as we wanted to reduce the risk of paraphrase ratings being influenced by adjective-noun combinations unfamiliar to the subjects. Furthermore, we excluded pairs with deverbal nouns (i.e., nouns derived from a verb) such as fast programmer, since an interpretation can be easily arrived at for these pairs by mapping the deverbal noun to its corresponding verb. A list of deverbal nouns was obtained from two dictionaries, CELEX (Burnage 1990) and NOMLEX (Macleod et al. 1998).

We used the model outlined in Section 3.1 to derive meaning paraphrases for the 90 adjective-noun combinations. We imposed no threshold on the frequencies $f(e, a)$ and $f(r e l, e, n)$. The frequency $f(e, a)$ was obtained by mapping the adjective to its corresponding adverb: the adjective good was mapped to the adverbs good and well, the adjective fast was mapped to the adverb fast, easy was mapped to easily, hard was mapped to hard, right to rightly and right, safe to safely and safe, slow to slowly and slow, and wrong to wrongly and wrong. The adverbial function of the adjective difficult is expressed only periphrastically (i.e., in a difficult manner, with difficulty). As a result we obtained the frequency $f($ difficult,$e)$ only on the basis of infinitival constructions (see the examples in (54)). We estimated the probability $P(a, n, r e l, e)$ for each adjectivenoun pair by varying both the terms $e$ and rel. We thus derived both subject-related and object-related paraphrases for each adjective-noun pair.

For each adjective-noun combination, the set of the derived meanings was again divided into three "probability bands" (high, medium, and low) of equal size, and one interpretation was selected from each band. The division into bands ensured that the experimental stimuli represented the model's behavior for likely and unlikely paraphrases. We performed separate divisions for object-related and subject-related paraphrases, resulting in a total of six interpretations for each adjective-noun combination, as we wanted to determine whether there were differences in the model's predictions with respect to the argument function (i.e., object or subject) and also because we wanted to compare experimentally derived adjective biases against model-derived biases. Example stimuli (with object-related interpretations only) are shown in Table 17 for each of the nine adjectives.

Our experimental design consisted of the factors adjective-noun pair (Pair), grammatical function (Func) and probability band (Band). The factor Pair included 90 adjective-noun combinations. The factor Func had two levels (subject and object), whereas the factor Band had three levels (high, medium, and low). This yielded a total of Pair $\times$ Func $\times$ Band $=90 \times 2 \times 3=540$ stimuli. The number of the stimuli was too large for subjects to judge in one experimental session. We limited the size of the design by selecting a total of 270 stimuli according to the following criteria: Our initial design created two sets of stimuli, 270 subject-related stimuli and 270 object-related stimuli. For each set of stimuli (i.e., object- and subject-related) we randomly selected five nouns for each of the nine adjectives, together with their corresponding interpretations in the three probability bands (high, medium, low). This yielded a total of Pair $\times$ Func $\times$ Band $=45 \times 2 \times 3=270$ stimuli. In this way, stimuli were created for each adjective in both subject-related and object-related interpretations.

As in Experiment 1, the stimuli were administered to two separate subject groups in order to limit the size of the experiment. Each group saw 135 stimuli consisting of interpretations for all adjective-noun pairs. For the first group five adjectives were 
Table 17

Randomly selected example stimuli with log-transformed probabilities derived by the model.

\begin{tabular}{lllllll}
\hline Adjective-noun & \multicolumn{2}{c}{ High } & \multicolumn{3}{c}{$\begin{array}{c}\text { Probability Band } \\
\text { Medium }\end{array}$} & \multicolumn{2}{c}{ Low } \\
\hline difficult customer & satisfy & -20.27 & help & -22.20 & drive & -22.64 \\
easy food & cook & -18.94 & introduce & -21.95 & finish & -23.15 \\
fast pig & catch & -23.98 & stop & -24.30 & use & -25.66 \\
good postcard & send & -20.17 & draw & -22.71 & look at & -23.34 \\
hard number & remember & -20.30 & use & -21.15 & create & -22.69 \\
right school & apply to & -19.92 & complain to & -21.48 & reach & -22.90 \\
safe drug & release & -22.24 & try & -23.38 & start & -25.56 \\
slow child & adopt & -19.90 & find & -22.50 & forget & -22.79 \\
wrong color & use & -21.78 & look for & -22.78 & look at & -24.89 \\
\hline
\end{tabular}

represented by object-related meanings only (difficult, easy, good, hard, slow); these adjectives were presented to the second group with subject-related interpretations only. Correspondingly, for the first group, four adjectives were represented by subject-related meanings only (safe, right, wrong, fast); the second group saw these adjectives with object-related interpretations.

Each experimental item consisted of an adjective-noun pair and a sentence paraphrasing its meaning. Paraphrases were created by the experimenters by converting the model's output to a simple phrase, usually a noun modified by a relative clause. A native speaker of English other than the authors was asked to confirm that the paraphrases were syntactically well-formed (items found syntactically odd were modified and retested). Example stimuli are shown in (55). A complete list of the experimental items is given in Appendix B.

(55) a. high: difficult customer a customer who is difficult to satisfy

b. medium: difficult customer

c. low: difficult customer a customer who is difficult to help a customer who is difficult to drive

(56) a. high: fast horse a horse that runs fast

b. medium: fast horse a horse that works fast

c. low: fast horse a horse that sees quickly

3.3.1.2 Procedure. The method used was magnitude estimation, with the same experimental protocol as in Experiment 1.

3.3.1.3 Instructions, Demographic Questionnaire, and Training Phase. The instructions were the same as in Experiment 1, with the exception that this time the subjects were asked to judge how well a sentence paraphrased a particular adjective-noun combination. The demographic questionnaire and the training phase were the same as in Experiment 1 .

3.3.1.4 Experimental Phase. Each subject group saw 135 metonymic sentences and their paraphrases. A modulus item from the medium probability band was provided (see 


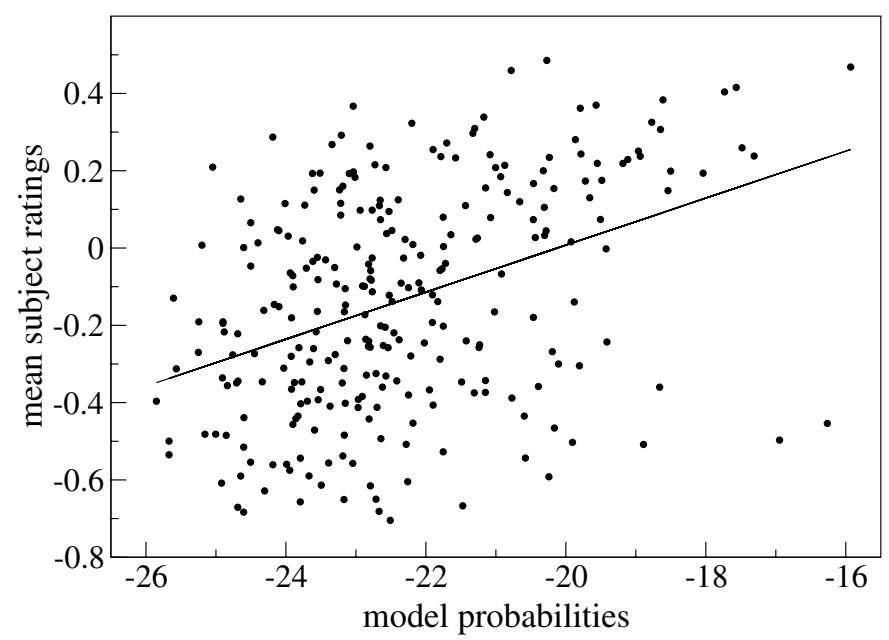

Figure 2

Correlation of elicited judgments and model-derived probabilities for metonymic adjective-noun pairs.

Appendix B). The modulus was the same for all subjects and remained on the screen the entire time the subject was completing the task. Subjects were assigned to groups at random, and a random stimulus order was generated for each subject.

3.3.1.5 Subjects. Sixty-five native speakers of English participated in the experiment. ${ }^{4}$ The subjects were recruited over the Internet by postings to relevant newsgroups and mailing lists. Participation was voluntary and unpaid.

The data of one subject were eliminated after inspection of his response times showed that he had not completed the experiment in a realistic time frame (average response time $<1000 \mathrm{~ms}$ ). The data of four subjects were excluded because they were non-native speakers of English.

This left 60 subjects for analysis. Of these, 54 subjects were right-handed, six lefthanded; 22 subjects were female, 38 male. The age of the subjects ranged from 18 to 54 years; the mean was 27.4 years.

\subsection{Results}

The data were normalized as in Experiment 1. We tested the hypothesis that paraphrases with high probabilities are perceived as better paraphrases than paraphrases assigned low probabilities by examining the degree to which the elicited judgments correlate with the probabilities derived by the model. As in Experiment 1, the data used for the judgments were the average of log-transformed and normalized subject ratings per experimental item. The comparison between model probabilities and the human judgments yielded a Pearson correlation coefficient of .40 $(p<.01, N=270)$. Figure 2 plots the relationship between judgments and model probabilities. Descriptive statistics for model probabilities and subject judgments are given in Appendix C.

In order to evaluate whether grammatical function has any effect on the relationship between model-derived meaning paraphrases and human judgments, we split the items into those that received a subject interpretation and those that received an

4 None of the participants of Experiment 1 took part in Experiment 4. 
object interpretation. A comparison between our model and human judgments yielded a correlation of $r=.53(p<.01, N=135)$ for object-related items and a correlation of $r=.21(p<.05, N=135)$ for subject-related items. Note that a weaker correlation is obtained for subject-related interpretations. One explanation for this weaker correlation could be the parser's performance; that is, the parser may be better at extracting verb-object tuples than verb-subject tuples. Another hypothesis (which we test below) is that most adjectives included in the experimental stimuli have an object bias, and therefore subject-related interpretations are generally less preferred than object-related ones.

Using leave-one-out resampling (see Section 2.3.2 for details), we calculated how well subjects agreed in their judgments concerning metonymic adjective-noun combinations. For the first group, the average intersubject agreement was .67 (Min $=.03$, $\operatorname{Max}=.82, \operatorname{StdDev}=.14)$, and for the second group it was $.65(\mathrm{Min}=.05, \mathrm{Max}=.82$, StdDev $=.14$ ).

The elicited judgments can be further used to derive the grammatical function preferences (i.e., subject or object) for a given adjective. In particular, we can determine the preferred interpretation for individual adjectives on the basis of the human data and then compare these preferences against the ones produced by our model. Argument preferences can be easily derived from the model's output by comparing subject-related and object-related paraphrases. For each adjective we gathered the subject- and object-related interpretations derived by the model and performed a one-way analysis of variance (ANOVA) in order to determine the significance of the grammatical function effect.

We interpret a significant effect as bias toward a particular grammatical function. We classify a particular adjective as object-biased if the mean of the model-derived probabilities for the object interpretation of that adjective is significantly larger than the mean for the subject interpretation; subject-biased adjectives are classified through a comparable procedure, whereas adjectives for which no effect of grammatical function is found are classified as equibiased. The effect of grammatical function was significant for the adjectives difficult $(F(1,1806)=8.06, p<.01)$, easy $(F(1,1511)=41.16, p<.01)$, hard $(F(1,1310)=57.67, p<.01)$, safe $(F(1,382)=5.42, p<.05)$, right $(F(1,2114)=9.85$, $p<.01)$, and fast $(F(1,92)=4.38, p<.05)$. The effect of grammatical function was not significant for the adjectives good $(F(1,741)=3.95, p=.10)$, slow $(F(1,759)=$ $5.30, p=.13)$, and wrong $(F(1,593)=1.66, p=.19)$. Table 18 shows the biases for the nine adjectives as derived by our model. A check mark next to a grammatical function indicates that its effect was significant in that particular instance, as well as the direction of the bias.

Ideally, we would like to elicit argument preferences from human subjects in a similar fashion. However, since it is impractical to elicit judgments experimentally for all paraphrases derived by the model, we will obtain argument preferences from the judgments based on the restricted set of experimental stimuli, under the assumption that they correspond to a wide range of model paraphrases (i.e., they correspond to a wide range of probabilities) and therefore are representative of the entire set of model-derived paraphrases. This assumption is justified by the fact that items were randomly chosen from the three probability bands (i.e., high, medium, low). For each adjective we gathered the elicited responses pertaining to subject- and object-related interpretations and performed an ANOVA.

The ANOVA indicated that the grammatical function effect was significant for the adjective difficult in both by-subjects (subscript 1) and by-items (subscript 2) analyses $\left(F_{1}(1,58)=17.98, p<.01 ; F_{2}(1,4)=53.72, p<.01\right)$, and for the adjective easy in the by-subjects analysis only $\left(F_{1}(1,58)=10, p<.01 ; F_{2}(1,4)=8.48, p=.44\right)$. No effect of 
Table 18

Log-transformed model-derived and subject-based argument preferences for polysemous adjectives.

\begin{tabular}{lccccccrr}
\hline Adjective & Model & Mean & StdDev & StdEr & Subjects & Mean & StdDev & StdEr \\
\hline difficult & $\sqrt{ }$ OBJ & -21.62 & 1.36 & .04 & $\sqrt{ }$ OBJ & .0745 & .3753 & .0685 \\
& SUBJ & -21.80 & 1.34 & .05 & SUBJ & -.2870 & .2777 & .0507 \\
\hline easy & $\sqrt{ }$ OBJ & -21.60 & 1.51 & .05 & $\sqrt{ }$ OBJ & .1033 & .3364 & .0614 \\
& SUBJ & -22.11 & 1.36 & .06 & SUBJ & -.1437 & .2308 & .0421 \\
\hline fast & OBJ & -24.20 & 1.27 & .13 & OBJ & -.3544 & .2914 & .0532 \\
& $\sqrt{ }$ SUBJ & -23.80 & 1.40 & .14 & $\sqrt{ }$ SUBJ & -.1543 & .4459 & .0814 \\
\hline good & OBJ & -22.12 & 1.28 & .06 & OBJ & -.0136 & .3898 & .0712 \\
& SUBJ & -22.27 & 1.10 & .07 & SUBJ & -.1563 & .2965 & .0541 \\
\hline hard & $\sqrt{ }$ OBJ & -21.69 & 1.53 & .06 & $\sqrt{ }$ OBJ & .0030 & .3381 & .0617 \\
& SUBJ & -22.12 & 1.35 & .06 & SUBJ & -.2543 & .2436 & .0445 \\
\hline \multirow{2}{*}{ right } & $\sqrt{ }$ OBJ & -21.65 & 1.36 & .04 & $\sqrt{ }$ OBJ & -.0054 & .2462 & .0450 \\
& SUBJ & -21.84 & 1.24 & .04 & SUBJ & -.2413 & .4424 & .0808 \\
\hline safe & OBJ & -22.75 & 1.48 & .10 & $\sqrt{ }$ OBJ & .0037 & .2524 & .0461 \\
& $\sqrt{ }$ SUBJ & -22.39 & 1.59 & .12 & SUBJ & -.3399 & .4269 & .0779 \\
\hline slow & OBJ & -22.49 & 1.53 & .08 & OBJ & -.3030 & .4797 & .0876 \\
& SUBJ & -22.32 & 1.50 & .07 & $\sqrt{ }$ SUBJ & -.0946 & .2357 & .0430 \\
\hline wrong & OBJ & -23.15 & 1.33 & .08 & $\sqrt{ }$ OBJ & -.0358 & .2477 & .0452 \\
& SUBJ & -23.29 & 1.30 & .08 & SUBJ & -.2356 & .3721 & .0679
\end{tabular}

grammatical function was found for good $\left(F_{1}(1,58)=2.55, p=.12 ; F_{2}(1,4)=1.01\right.$, $p=.37)$. The effect of grammatical function was significant for the adjective hard in the by-subjects analysis only $\left(F_{1}(1,58)=11.436, p<.01 ; F_{2}(1,4)=2.84, p=.17\right)$, whereas for the adjective slow the effect was significant both by subjects and by items $\left(F_{1}(1,58)=4.56, p<.05 ; F_{2}(1,4)=6.94, p=.058\right)$. For safe and right the main effect was significant in both by-subjects and by-items analyses $\left(F_{1}(1,58)=14.4, p<.0005\right.$; $F_{2}(1,4)=17.76, p<.05$, and $F_{1}(1,58)=6.51, p<.05 ; F_{2}(1,4)=15.22, p=.018$, respectively). The effect of grammatical function was significant for wrong and fast only by subjects $\left(F_{1}(1,58)=5.99, p=.05 ; F_{2}(1,4)=4.54, p=.10\right.$, and $F_{1}(1,58)=4.23, p=.05$; $\left.F_{2}(1,4)=4.43, p=.10\right)$. The biases for these adjectives are shown in Table 18. Check marks again indicate instances in which the grammatical function effect was significant (as determined from the by-subjects analyses), as well as the direction of the bias.

We expect a valid model to assign on average higher probabilities to object-related interpretations and lower probabilities to subject-related interpretations for an objectbiased adjective; accordingly, we expect the model to assign on average higher probabilities to subject-related interpretations for subject-biased adjectives. Comparison of the biases derived from the model with ones derived from the elicited judgments shows that the model and the humans are in agreement for all adjectives but slow, wrong, and safe. On the basis of human judgments, slow has a subject bias and wrong has an object bias (see Table 18). Although the model could not reproduce this result, there was a tendency in the right direction.

Note that in our correlation analysis reported above, the elicited judgments were compared against model-derived paraphrases without taking argument preferences 
into account. We would expect a valid model to produce intuitive meanings at least for the interpretation that a given adjective favors. We further examined the model's behavior by performing separate correlation analyses for preferred and dispreferred biases as determined previously by the ANOVAs conducted for each adjective (see Table 18). Since the adjective good was equibiased, we included both biases (i.e., objectrelated and subject-related) in both correlation analyses for that adjective. The comparison between our model and the human judgments yielded a Pearson correlation coefficient of $.52(p<.01, N=150)$ for the preferred interpretations and a correlation of $.23(p<.01, N=150)$ for the dispreferred interpretations. The result indicates that our model is particularly good at deriving meanings corresponding to the argument bias for a given adjective. However, the dispreferred interpretations also correlate significantly with human judgments, which suggests that the model derives plausible interpretations even in cases in which the argument bias is overridden.

In sum, the correlation analysis supports the claim that adjective-noun paraphrases with high probability are judged more plausible than those with low probability. It also suggests that the meaning preference ordering produced by the model is intuitively correct, since subjects' perception of likely and unlikely meanings correlates with the probabilities assigned by the model.

The probabilistic model evaluated here explicitly takes adjective/adverb and verb co-occurrences into account. However, one could derive meanings for polysemous adjective-noun combinations by concentrating solely on verb-noun relations, ignoring thus the adjective/adverb and verb dependencies. For example, in order to interpret the combination easy problem, we could simply take into account the types of activities that are related to problems (i.e., solving them, giving them, etc.). This simplification is consistent with Pustejovsky's (1995) claim that polysemous adjectives like easy are predicates, modifying some aspect of the head noun and more specifically the events associated with the noun. A naive baseline model would be one that simply takes into account the number of times the noun in the adjective-noun pair acts as the subject or object of a given verb, ignoring the adjective completely. This raises the question of how well such a naive model would perform at deriving meaning paraphrases for polysemous adjective-noun combinations.

In the following section we present such a naive model of adjective-noun polysemy. We compare the model's predictions against the elicited judgments. Using correlation analysis we attempt to determine whether the naive model can provide an intuitively plausible ranking of meanings (i.e., whether perceived likely and unlikely meanings are given high and low probabilities, respectively). We further compare the naive model to our initial model (see Section 3.1) and discuss the differences between them.

\subsection{Experiment 5: Comparison against Naive Baseline}

3.5.1 Naive Baseline Model. Given an adjective-noun combination, we are interested in finding the events most closely associated with the noun modified by the adjective. In other words we are interested in the verbs whose object or subject is the noun appearing in the adjective-noun combination. This can be simply expressed as $P(e \mid r e l, n)$, the conditional probability of a verb $e$ given an argument-noun relation $r e l, n$ :

$$
P(e \mid r e l, n)=\frac{f(e, r e l, n)}{f(r e l, n)}
$$

The model in (57) assumes that the meaning of an adjective-noun combination is independent of the adjective in question. Consider, for example, the adjective-noun 
pair fast plane. We need to find the verbs $e$ and the argument relation rel that maximize the probability $P(e \mid r e l$, plane $)$. In the case of fast plane, the verb that is most frequently associated with planes is fly (see Table 13). Note that this model will come up with the same probabilities for fast plane and wrong plane, since it does not take the identity of the modifying adjective into account. We estimated the frequencies $f(e, r e l, n)$ and $f(r e l, n)$ from verb-object and verb-subject tuples extracted from the BNC using Cass (Abney 1996) (see Section 2.2 for details on the extraction and filtering of the argument tuples).

\subsection{Results}

Using the naive model we calculated the meaning probability for each of the 270 stimuli included in Experiment 4 and explored the linear relationship between the elicited judgments and the naive baseline model through correlation analysis. The comparison yielded a Pearson correlation coefficient of $.25(p<.01, N=270)$. Recall that we obtained a correlation of $.40(p<.01, N=270)$ when comparing our original model to the human judgments. Not surprisingly the two models are intercorrelated $(r=.38, p<.01, N=270)$. An important question is whether the difference between the two correlation coefficients $(r=.40$ and $r=.25)$ is due to chance. A one-tailed $t$ test revealed that the difference between them was significant $(t(267)=2.42, p<.01)$. This means that our original model (see Section 3.1) performs reliably better than the naive baseline at deriving interpretations for metonymic adjective-noun combinations.

We further compared the naive baseline model and the human judgments separately for subject-related and object-related items. The comparison yielded a correlation of $r=.29(p<.01, N=135)$ for object interpretations. Recall that our original model yielded a correlation coefficient of .53 (see Section 3.4). A one-tailed $t$-test revealed that the two correlation coefficients were significantly different $(t(132)=3.03, p<.01)$. No correlation was found for the naive model when compared against elicited subject interpretations $(r=.09, p=.28, N=135)$.

\subsection{Discussion}

We have demonstrated that the meanings acquired by our probabilistic model correlate reliably with human intuitions. Our model not only acquires clusters of meanings (following Vendler's (1968) insight) but furthermore can be used to obtain a tripartite distinction of adjectives depending on the type of paraphrase they prefer: subjectbiased adjectives tend to modify nouns that act as subjects of the paraphrasing verb, and object-biased adjectives tend to modify nouns that act as objects of the paraphrasing verb, whereas equibiased adjectives display no preference for either argument role. A comparison between the argument preferences produced by the model and human intuitions revealed that most of the adjectives we examined (six out of nine) display a preference for an object interpretation (see Table 18), two adjectives are subject-biased (i.e., fast, slow) and one adjective is equibiased (i.e., good).

We rigorously evaluated ${ }^{5}$ the results of our model by eliciting paraphrase judgments from subjects naive to linguistic theory. Comparison between our model and human judgments yielded a reliable correlation of .40 when the upper bound for the task (i.e., intersubject agreement) is approximately .65. We have demonstrated that a naive baseline model that interprets adjective-noun combinations by focusing solely on the events associated with the noun is outperformed by a more detailed model that

\footnotetext{
5 We have not compared the model's predictions against norming data for adjectival metonymies primarily because such data were not available to us. To our knowledge McElree et al.'s (2001) study is the only example of a norming study on logical metonymy; however, it concentrates only on verbs.
} 
considers not only verb-argument relations but also adjective-verb and adverb-verb dependencies.

Although the events associated with the different nouns are crucially important for the meaning of polysemous adjective-noun combinations, it seems that more detailed linguistic knowledge is needed in order to produce intuitively plausible interpretations. This is by no means surprising. As a simple example, consider the adjectivenoun pair fast horse. A variety of events are associated with the noun horse, yet only a subset of those are likely to occur fast. The three most likely interpretations for fast horse according to the naive model are a horse that needs something fast, a horse that gets something fast, and a horse that does something fast. A model that uses information about verb-adjective or verb-adverb dependencies provides a more plausible ranking: a fast horse is a horse that runs, learns, or goes fast. A similar situation arises when one considers the pair careful scientist. According to the naive model, a careful scientist is more likely to believe, say, or make something carefully. However, none of these events is particularly associated with the adjective careful.

Although our experiments on adjectival logical metonymy revealed a reliable correlation between the model's ranking and human intuitions, the fit between model probabilities and elicited judgments was lower for metonymic adjectives $(r=.40)$ than for metonymic verbs $(r=.64)$. One explanation for this lower degree of fit for adjectives is that the semantic restrictions that adjectives impose on the nouns with which they combine appear to be less strict than the ones imposed by verbs (consider, for example, the adjective good, which can combine with nearly any noun). A consequence of this is that metonymic adjectives seem to allow a wider range of interpretations than verbs. This means that there will be a larger variation in subjects' responses to the generated model paraphrases when it comes to adjectives than when it comes to verbs, thus affecting the linear relationship between our model and the elicited judgments. Our hypothesis is further supported by the intersubject agreement, which is lower for metonymic adjectives than for verbs (.65 versus .74). As explained in the previous sections, our model does not take into account the wider context within which an adjective-noun combination is found. Precisely because of the ease with which some adjectives combine with practically any noun, it may be the case that more information (i.e., context) is needed in order to obtain intuitively plausible interpretations for metonymic adjectives. The model presented here can be extended to incorporate contextual information (e.g., intra- and extrasentential information), but we leave this to future work.

\section{General Discussion}

In this article we have focused on the automatic interpretation of logical metonymy. We have shown how meaning paraphrases for metonymic expressions can be acquired from a large corpus and have provided a probabilistic model that derives a preference ordering on the set of possible meanings in an unsupervised manner, without relying on the availability of a disambiguated corpus. The proposed approach utilizes surface syntactic analysis and distributional information that can be gleaned from a corpus while exploiting correspondences between surface cues and meaning.

Our probabilistic model reflects linguistic observations about the nature of metonymic constructions: It predicts variation in interpretation for different verbs and adjectives with respect to the noun for which they select and is faithful to Vendler's (1968) claim that metonymic expressions are usually interpreted by a cluster of meanings instead of a single meaning. This contrasts with Pustejovsky's (1995) approach, which typically assigns a single reading to the metonymic construction, and with the account 
put forward by Copestake and Briscoe (1995), which assigns one interpretation conventionally, albeit a default interpretation-in fact this interpretation might also be of quite a general type (e.g., the event argument to enjoy in enjoy the pebble can be assigned the general type act-on). Our model provides plausible alternatives to the default, and it augments the general semantic type in the interpretation that's assigned by these theories with a plausible range of more specific values, although, in contrast to the hybrid model of interpretation described in Copestake and Lascarides (1997), it does not predict the contexts in which a statistically dispreferred interpretation is the correct one.

Our approach can be viewed as complementary to linguistic theory: Although our model does not identify odd metonymies in the way that a rule-based model might (we return to this in Section 4.1), it does automatically derive a ranking of meanings, thus distinguishing likely from unlikely interpretations. Even if linguistic theory is able to enumerate all possible interpretations for a given adjective (note that in the case of polysemous adjectives, we would have to take into account all nouns or noun classes that the adjective could possibly modify), in most cases it does not indicate which ones are likely and which ones are not. Our model fares well on both tasks. It recasts the problem of logical metonymy in a probabilistic framework and derives a large number of interpretations not readily available from linguistic introspection. The information acquired from the corpus can be also used to quantify the argument preferences of metonymic adjectives. These are only implicit in the lexical semantics literature, in which certain adjectives are exclusively given a verb-subject or verb-object interpretation.

\subsection{Limitations and Extensions}

We chose to model metonymic constructions and their meanings as joint distributions of interdependent linguistic events (e.g., verb-argument relations, verb-adverb modification). Although linguistically informed, our approach relies on approximations and simplifying assumptions, partly motivated by the absence of corpora explicitly annotated with metonymic interpretations. We generate meaning paraphrases for metonymic constructions solely from co-occurrence data without taking advantage of taxonomic information. Despite the simplicity of this approach and its portability to languages for which lexical semantic resources may not be available, there are certain regularities about the derived interpretations that our model fails to detect.

Consider the interpretations produced for begin song, repeated here from Table 7: sing, rehearse, write, hum, and play. Our model fails to capture the close correspondence for some of these meanings. For example, hum and sing are sound emission verbs; they additionally entail the performance or execution of the song. The verbs rehearse and play capture only the performance aspect and can be thus considered supertypes of hum and sing (one can play or rehearse a song by humming it, singing it, drumming it, whistling it, etc.). The verb write, on the other hand, is neither a performance nor a sound emission verb; it has to do with communication and creation. Another example is comfortable chair, for which the model generates sink into, sit on, lounge in, relax in, and nestle in (see Table 15). The verbs sink into, sit on, lounge in, and nestle in describe the position one assumes when sitting in the chair, whereas relax in refers to the state of the person in the chair. There is no notion of semantic proximity built into the model, and correspondences among semantically related interpretations are not automatically recognized.

An alternative to the knowledge-free approach advocated here is to use taxonomic information to obtain some degree of generalization over the acquired interpretations. Semantic classifications such as WordNet (Miller et al., 1990) or that in Levin (1993) can be used to group the obtained verbs into semantically coherent classes. Further- 
more, the WordNet semantic hierarchy can be used to estimate directly probabilities involving either nouns (e.g., $P(r e l \mid e, n), P(o \mid e)$ ) or verbs (e.g., $P(v \mid e), P(a \mid e)$ ). Probabilities can be defined in terms of senses from a semantic hierarchy by exploiting the fact that the senses can be grouped into classes consisting of semantically similar senses (Resnik 1993; Clark and Weir 2001; McCarthy 2000; Li and Abe 1998). So the probability $P($ book $\mid$ read $)$ can be estimated by taking into account nouns that belong to the same semantic class as book and can be read (e.g., journals, novels, scripts) or by focusing on verbs that are semantically related to read (e.g., interpret, communicate, understand). Note that estimation of probabilities over classes rather than words can effectively overcome data sparseness and potentially lead to better probability estimates. Currently our models cannot estimate probabilities for word combinations unseen in the corpus, and WordNet could be used for re-creating the frequencies of these combinations (Lapata, Keller, and McDonald 2001; Clark and Weir 2001). However, part of our aim here was to investigate whether it is at all possible to generate interpretations for metonymic constructions without the use of prior knowledge bases that might bias the acquisition process in uncontrolled and idiosyncratic ways.

A related issue is the fact that our models are ignorant about the potentially different senses of the noun in the metonymic construction. For example, the combination fast plane may be a fast aircraft, or a fast tool, or a fast geometrical plane. Our model derives meanings related to all three senses of the noun plane. For example, a fast plane is not only a plane (i.e., an aircraft) that flies, lands, or travels quickly, but also a plane (i.e., a surface) that transposes or rotates quickly and a plane (i.e., a tool) that smoothes something quickly. However, more paraphrases are derived for the "aircraft" sense of plane; these paraphrases also receive a higher ranking. This is not surprising, since the number of verbs related to the "aircraft" sense of plane are more frequent than the verbs related to the other two senses. In contrast to fast plane, however, efficient plane should probably bias toward the "tool" sense of plane, even though the "aircraft" sense is more frequent in the corpus. One could make the model sensitive to this by investigating the synonyms for the various senses of plane; moreover the "tool" sense bias of efficient plane could also be inferred on the basis that efficient plane co-occurs with different verbs from fast plane. There are also cases in which a model-derived paraphrase does not provide disambiguation clues with respect to the meaning of the noun. Consider the adjective-noun combination fast game. The model comes up with the paraphrases game that runs fast and game that goes fast. Both paraphrases may well refer to either the "contest," "activity," or "prey" sense of game. Note finally that our model can be made sensitive to word sense distinctions by taking into account noun classes rather than word forms; this modification would allow us to apply the model to word sense-disambiguated metonymic expressions.

In this article we have focused on the automatic interpretation of logical metonymy without explicitly dealing with the recognition of verbs or adjectives undergoing logical metonymy. In default of the latter study, which we plan for the future, we sketch here briefly how our proposal can be extended to recognizing logical metonymies. A very simple approach would be to use the proposed model to generate interpretations for metonymic and nonmetonymic constructions. The derived paraphrases and the range of their probabilities could be then used to quantify the degree of "metonymic-ness" of a given verb or adjective. One would expect that a larger number of paraphrases would be generated for verbs or adjectives for which logical metonymy is possible. We tested this hypothesis using a metonymic verb (i.e., enjoy) and a nonmetonymic one (i.e., play). Enjoy is attested 5,344 times in the BNC in a verb-object relation, whereas play is attested 12,597 times (again these numbers are based on information extracted using Cass (Abney 1996)). Using the model presented in Section 2.1 we generated 
Table 19

Model-derived paraphrases for odd metonymies, ranked in order of likelihood.

\begin{tabular}{llllllll}
\hline \multicolumn{2}{c}{ begin dictionary } & \multicolumn{2}{c}{ begin rock } & & \multicolumn{2}{c}{ begin keyboard } & \multicolumn{2}{c}{ begin highway } \\
\hline compile & -19.32 & crunch across & -18.09 & use & -20.11 & obstruct & -20.40 \\
flick through & -19.59 & climb & -18.39 & play & -20.44 & regain & -20.79 \\
use & -19.80 & run towards & -18.70 & operate & -20.56 & build & -20.80 \\
publish & -20.34 & percolate through & -18.78 & assemble & -20.78 & use & -20.81 \\
advance & -20.39 & dissolve & -19.37 & tune & -20.80 & detach & -20.82
\end{tabular}

meaning paraphrases for all verb-object tuples found for enjoy and play. The model generated 44,701 paraphrases for enjoy and 9,741 for play. Comparison between the probabilities assigned to the interpretations for enjoy and play revealed that the paraphrases obtained for enjoy were on average more likely (mean $=-23.53$, $\min =-27.02$, $\max =-15.32$ ) than those discovered for play (mean $=-24.67$, $\min =-28.25$, $\max$ $=-17.24$ ). The difference was statistically significant (using an independent-samples $t$-test; $t(54,440)=2.505, p<.01)$. This result indicates that enjoy is more likely to undergo logical metonymy than play. Another potential indicator of metonymic use is the likelihood that a given verb or adjective will be found in a certain syntactic construction. Consider the adjective blue, for which our model (see Section 3.1) does not generate any meaning paraphrases, presumably because blue is not attested as a verb modifier (in contrast to adjectives like easy or fast).

Such an approach could potentially predict differences in productivity among metonymic verbs. One would expect the metonymic uses of attempt, for example, to be much less productive than the metonymic uses of enjoy and begin. One could conceivably predict this on the basis of the frequency and diversity of NPs in attempt NP constructions that are attested in the corpus, compared with those for enjoy NP and begin NP. Furthermore, the model generates paraphrases for attempt that are on average less likely in comparison to those generated for enjoy or begin. However, we leave this for future work.

As argued in Section 2.1, our model cannot distinguish between well-formed and odd metonymic constructions; in fact, it will generally provide meaning paraphrases even for combinations that are deemed by native speakers to be odd. Consider the examples in (58) and their interpretations in Table 19. In general the paraphrases generated for problematic data are of worse quality than those produced for well-formed metonymies. In most cases the model will generate unavailable interpretations (see begin highway, begin keyboard). Consider, however, the pair begin rock. Pustejovsky (1995) observes that although there is no generally available interpretation for a sentence like Mary began the rock, because of what we understand begin to require of its argument and our knowledge of what rocks are and what you can do to them, as speakers and hearers we tend to accommodate information into the context so as to interpret otherwise ill-formed expressions. Our model generates meaning paraphrases that are relatively plausible assuming different pragmatic contexts for begin rock. One can begin climbing or running towards a rock. Someone's footsteps can crunch across a frozen rock, a material can percolate through a rock, and rain water can dissolve a rock.

(58) a. ?John began the dictionary.

b. ?Mary began the rock.

c. *John began a keyboard.

d. *John began the highway. 
Table 20

Descriptives for odd and well-formed metonymies.

\begin{tabular}{lcccc}
\hline Well-formed & $\boldsymbol{N}$ & Mean & StdDev & StdErr \\
\hline begin book & 534 & -21.54 & 1.515 & .066 \\
begin cigarette & 104 & -21.83 & 1.613 & .158 \\
begin coffee & 104 & -22.03 & 1.626 & .159 \\
begin story & 381 & -21.73 & 1.493 & .076 \\
easy problem & 358 & -21.14 & 1.606 & .085 \\
\hline Odd & $N$ & Mean & StdDev & StdErr \\
\hline begin dictionary & 76 & -22.48 & 1.440 & .165 \\
begin keyboard & 50 & -22.40 & 1.337 & .189 \\
begin rock & 50 & -23.55 & 1.376 & .193 \\
begin highway & 37 & -22.52 & 1.337 & .219 \\
easy programmer & 49 & -23.23 & 1.289 & .184
\end{tabular}

Despite the fact that the model does not recognize odd metonymies, one would expect low probabilities to be assigned to ungrammatical constructions. Table 20 reports some descriptive statistics on well-formed (top half) and odd (bottom half) metonymies taken from the lexical semantics literature (Verspoor 1997; Pustejovsky 1995). A higher number of interpretations is generated for well-formed metonymies. Using an independent-samples $t$-test, we can compare the differences in the probabilities assigned to the two types of metonymies. Take, for example, begin dictionary: the average probability of its interpretations is lower than those for begin book $(t(608)=5.07$, $p<.01)$, begin cigarette $(t(178)=2.77, p<.01)$, begin coffee $(t(178)=2.1, p<.05)$, and begin story $(t(455)=4.1, p<.01)$. Similar results are obtained when comparing begin keyboard against the well-formed metonymies in Table 19: the probability of its interpretations is on average lower than those assigned to begin book, $(t(582)=3.87$, $p<.01)$, begin cigarette $(t(152)=2.15, p<.01)$, and begin story $(t(429)=3.02, p<.01)$. The difference between begin coffee and begin keyboard is not statistically significant $(t(152)=1.39, p=0.167)$. However, the mean for begin coffee is slightly higher than that for begin keyboard. Although here we focus on verbs, similar comparisons can be applied to adjectives. As shown in Table 20, the probabilities for easy programmer are on average lower than those for easy problem $(t(405)=8.74, p<.01)$.

Finally, recall from Section 2.1 that on the basis of Gricean reasoning, one would expect to find in a corpus well-formed metonymies more often than their paraphrases (see Tables 1-3). Following this line of reasoning, one might expect for conventionally odd metonymies the opposite situation, that is, to find the paraphrases more often than the metonymies proper. We tested this hypothesis for some examples cited in the literature (Verspoor 1997; Pustejovsky 1995) by examining whether paraphrases corresponding to odd metonymies are attested in the BNC as VP complements. We found plausible paraphrases in the BNC for almost all verb-noun pairs illustrated in Table 21. This suggests that the corpus data relating to the odd and well-formed examples are largely compliant with the Gricean predictions. Corpus co-occurrences of verb-noun combinations and their paraphrases could be exploited in creating a system aimed at quantifying the grammaticality of metonymic expressions. However, it is beyond the scope of the present study to develop such a system. 
Table 21

BNC frequencies for odd metonymic expressions.

\begin{tabular}{lcr}
\hline \multicolumn{1}{c}{ Odd } & begin $\mathbf{N P}$ & begin V-ing $\mathbf{~ N P}$ \\
\hline begin chair & 0 & 9 \\
begin tunnel & 0 & 4 \\
begin keyboard & 0 & 0 \\
begin tree & 1 & 13 \\
begin highway & 0 & 2 \\
begin film & 0 & 7 \\
begin nail & 0 & 4 \\
begin door & 0 & 18 \\
begin dictionary & 0 & 3 \\
begin rock & 0 & 17 \\
& & \\
\hline
\end{tabular}

Table 22

Five most likely interpretations for good author and good language.

\begin{tabular}{llllll}
\hline & good author & \multicolumn{3}{c}{ good language } \\
\hline write & SUBJ & -21.81 & use & OBJ & -17.39 \\
work & SUBJ & -21.97 & verse in & OBJ & -17.89 \\
describe & SUBJ & -22.03 & speak & OBJ & -18.32 \\
know & OBJ & -22.05 & know & OBJ & -19.18 \\
engage with & OBJ & -22.23 & learn & OBJ & -19.36
\end{tabular}

\subsection{Relevance for NLP Applications}

The meaning paraphrases discovered by our model could be potentially useful for a variety of NLP tasks. One obvious application is natural language generation. For example, a generator that has knowledge of the fact that fast plane corresponds to $a$ plane that flies fast can exploit this information either to render the text shorter (in cases in which the input representation is a sentence) or longer (in cases in which the input representation is an adjective-noun pair). Information retrieval is another relevant application. Consider a search engine faced with the query fast plane. Presumably one would not like to obtain information about planes in general or about planes that go down or burn fast, but rather about planes that fly or travel fast. So knowledge about the most likely interpretations of fast plane could help rank relevant documents before nonrelevant ones or restrict the number of documents retrieved.

Note that in the case of adjectives, it is not just the paraphrase, but also the grammatical function, that needs to be determined. How to render an adjective-noun combination with an object- or subject-related paraphrase can be worked out by computing the biases discussed in Section 3.4. So if we know that fast has a subject bias, we can concentrate only on the subject-related interpretations. The choice of grammatical function is less straightforward in the case of equibiased adjectives. In fact, it is possible that the interpretation for a particular adjective varies depending on the noun it modifies. For example, a good author writes well, whereas a good language is good to learn, hear, or study. A simple way to address this is to select the interpretations with the highest probability. For good author and good language, the five most likely interpretations (and their grammatical functions) according to the model (see Section 3.3) are given in Table 22. As can be seen from the table, subject-related interpretations are ranked higher for good author; the opposite is true for good language. Another possibil- 
ity for determining the grammatical function for equibiased adjectives is to compare verb-object and verb-subject interpretations directly for a particular adjective-noun combination. As an example, consider the following. For good author, the model produces 107 object-related paraphrases and 199 subject-related ones. Furthermore, the subject-related probabilities are on average higher than the object-related ones, and the difference is statistically significant (using a one-tailed $t$-test, $t(304)=3.26, p<.01$ ). For good language there are 253 object-related paraphrases and 180 subject-related ones. The former are assigned higher probabilities than the latter, and the difference is statistically significant $(t(431)=3.80, p<.01)$.

Machine translation is another related application. A logical metonymy may be acceptable in a source language but unacceptable in the target language. Consider the example in (59): Its direct translation into German produces a semantically unacceptable sentence (see (60a)). In this case we need to spell out the metonymy in order to obtain an acceptable translation for German, and our model can be used to provide the missing information by generating meaning paraphrases. Under such an approach, we would not translate (59) directly, but one of its paraphrases (see (60b) and (60c)).

Peter attempted the peak.

(60) a. Peter hat den Gipfel versucht.

Peter has the peak attempted

'Peter attempted the peak.'

b. Peter hat den Gipfel zu besteigen versucht.

Peter has the peak to climb attempted

'Peter attempted to climb the peak.'

c. Peter hat den Gipfel zu erreichen versucht.

Peter has the peak to reach attempted

'Peter attempted to reach the peak.'

\section{Related Work}

In contrast to the extensive theoretical literature on the topic of logical metonymy, little attention has been paid to the phenomenon from an empirical perspective. Briscoe, Copestake, and Boguraev (1990) and Verspoor (1997) undertake a manual analysis of logical metonymies found in naturally occurring text. Their results show that logical metonymy is a relatively widespread phenomenon and that most metonymic examples can be interpreted on the basis of the head noun's qualia structure, assuming a theoretical framework similar to Pustejovsky's (1991). Verspoor's (1997) analysis of the metonymic verbs begin and finish demonstrates that context plays a relatively small role in the interpretation of these verbs: $95.0 \%$ of the logical metonymies for begin and $95.6 \%$ of the logical metonymies for finish can be resolved on the basis of information provided by the noun for which the verb selects. Briscoe, Copestake, and Boguraev's (1990) work further suggests ways of acquiring qualia structures for nouns by combining information extracted from machine-readable dictionaries and corpora. Our probabilistic formulation of logical metonymy allows us to discover interpretations for metonymic constructions without presupposing the existence of qualia structures. In fact, we show that a simple statistical learner in combination with a shallow syntactic analyzer yields relatively intuitive results, considering the simplifications and approximations in the system. 
Perhaps more relevant to the work presented here are previous approaches to the automatic interpretation of general metonymy (Lakoff and Johnson 1980; Nunberg 1995). This is slightly different from logical metonymy, in that the examples aren't usually analyzed in terms of semantic type coercion. But the phenomena are closely related. Generally speaking an expression $A$ is considered a metonymy if $A$ deviates from its literal denotation in that it stands for an entity $B$ that is not expressed explicitly but is conceptually related to $A$ via a contiguity relation $r$ (Markert and Hahn 1997). A typical example of general metonymy is given in (61): in (61a) the bottle stands for its content (i.e., the liquid in the bottle), and in (61b) Shakespeare stands for his works. The contiguity relation $r$ between the bottle and its liquid is Container for Contents; for Shakespeare and his works the contiguity relation is Producer for Product.

(61) a. Denise drank the bottle.

b. Peter read Shakespeare.

Previous approaches to processing metonymy typically rely heavily on the availability of manually constructed knowledge bases or semantic networks (Fass 1991; Inverson and Helmreich 1992; Bouaud, Bachimont, and Zweigenbaum 1996; Hobbs et al. 1993). Furthermore, most implementations either contain no evaluation (Fass 1991; Inverson and Helmreich 1992; Hobbs et al. 1993) or report results on the development data (Bouaud, Bachimont, and Zweigenbaum 1996).

The approach put forward by Utiyama, Murata, and Isahara (2000) is perhaps the most comparable to our own work. Utiyama et al. describe a statistical approach to the interpretation of general metonymies for Japanese. Utiyama et al.'s algorithm interprets verb-object metonymies by generating the entities for which the object stands. These entities are ranked using a statistical measure. Given an expression like (62), nouns related to Shakespeare are extracted from the corpus (e.g., si 'poem', tyosyo 'writings', sakuhin 'works') and ranked according to their likelihood. Two types of syntactic relations are used as cues for the interpretation of metonymic expressions: (1) the noun phrase $A$ no $B$, roughly corresponding to the English $B$ of $A$, where $A$ is the noun figuring in the metonymic expression (e.g., Shakespeare in (62)) and $B$ is the noun it stands for (e.g., sakuhin 'works'), and (2) nouns co-occurring with the target noun (e.g., Shakespeare) within the target sentence.

$$
\begin{aligned}
& \text { Shakespeare wo yomu } \\
& \text { Shakespeare ACC read } \\
& \text { 'read Shakespeare' }
\end{aligned}
$$

Given a metonymy of the form $A R V$, the appropriateness of a noun $B$ as an interpretation of $A$ is defined as

$$
L_{Q}(B \mid A, R, V)=\frac{P(B \mid A, Q) P(R, V \mid B)}{P(R, V)}
$$

where $V$ is the verb in the metonymic expression, $A$ is its object, $R$ is $A^{\prime}$ s case marker (e.g., wo (accusative)), $B$ is the noun $A$ stands for, and $Q$ is the relation $Q$ bears to $A$ (e.g., no). The probabilities in (63) are estimated from a large, morphologically analyzed Japanese corpus of newspaper texts (approximately 153 million words). A Japanese thesaurus is used for the estimation of the term $P(R, V \mid B)$ when the frequency $f(R, V, B)$ is zero (see Utiyama, Murata, and Isahara (2000) for the derivation 
and estimation of (63)). Utiyama et al.'s approach is tested on 75 metonymies taken from the literature. It achieves a precision of $70.6 \%$ as measured by one of the authors according to the following criterion: A metonymic interpretation was considered correct if it made sense in some context.

Our approach is conceptually similar to that of Utiyama, Murata, and Isahara (2000). Metonymies are interpreted using corpora as the inventory of the missing information. In contrast to Utiyama et al., we use no information external to the corpus (e.g., a thesaurus); sparse-data problems are tackled via independence assumptions, and syntactic information is obtained through shallow text analysis (Utiyama, Murata, and Isahara (2000) rely on morphological analysis to provide cues for syntactic information). The most striking difference, however, between our work and Utiyama et al.'s is methodological. Their evaluation is subjective and limited to examples taken from the literature. The appropriateness of their statistical measure (see (63)) is not explored, and it is not clear whether it can derive an intuitively plausible ranking of interpretations or whether it can extend to examples found in naturally occurring text. We test our probabilistic formulation of logical metonymy against a variety of examples taken from the corpus, and the derived interpretations are evaluated objectively using standard experimental methodology. Furthermore, the appropriateness of the proposed model is evaluated via comparisons to a naive baseline.

\section{Conclusions}

In this article we proposed a statistical approach to logical metonymy. We acquired the meanings of metonymic constructions from a large corpus and introduced a probabilistic model that provides a ranking on the set of possible interpretations. We identified semantic information automatically by exploiting the consistent correspondences between surface syntactic cues and meaning.

We evaluated our results against paraphrase judgments elicited experimentally from subjects naive to linguistic theory and showed that the model's ranking of meanings correlates reliably with human intuitions. Comparison between our model and human judgments yields a reliable correlation of .64 for verb-noun combinations and .40 for adjective-noun pairs. Furthermore, our model performs reliably better than a naive baseline model, which achieves only a correlation of .42 in the case of verbs and .25 in the case of adjectives.

Our approach combined insights from linguistic theory (i.e., Pustejovsky's (1995) theory of qualia structure and Vendler's (1968) observations) with corpus-based acquisition techniques, probabilistic modeling, and experimental evaluation. Our results empirically tested the validity of linguistic generalizations and extended their coverage. Furthermore, in agreement with other lexical acquisition studies (Merlo and Stevenson 2001; Barzilay and McKeown 2001; Siegel and McKeown 2000; Light 1996; McCarthy 2000; Rooth et al. 1999), we showed that it is possible to extract semantic information from corpora even if they are not semantically annotated in any way.

\section{Appendix A. The WebExp Software Package}

As part of the evaluation of the probabilistic models presented in this article, we conducted two psycholinguistic experiments. These experiments were administered 
using WebExp (Keller, Corley, and Scheepers 2001), a software package designed for conducting psycholinguistic studies over the Web. ${ }^{6}$

WebExp is a set of Java classes for conducting psycholinguistic experiments over the World Wide Web. The software consists of two modules: the WebExp server, which is a stand-alone Java application, and the WebExp client, which is implemented as a Java applet. The server application runs on the Web server that hosts the experiment and waits for client applets to connect to it. It issues experimental materials to clients and records participants' responses. The client applet is typically embedded into a Web page that contains the instructions for the experiment. When a participant starts the experiment, the WebExp client will download the experimental materials from the WebExp server and administer them to the participant. After the experiment is completed, it will send the participants' responses to the server, along with other participant-specific data.

As Java is a full-fledged programming language, it gives the Web designer maximal control over the interactive features of a Web site. WebExp makes use of this flexibility to keep the experimental procedure as constant as possible across participants. An important aspect is that the sequence in which the experimental items are administered is fixed for each participant: The participant does not have the ability to go back to previous stimuli and to inspect or change previous responses. (If the participant hits the Back button on the browser, the experiment will terminate.) WebExp also provides timings of participant responses by measuring the response onset time and the completion time for each answer. The studies reported in this article make no direct use of these timings. Nevertheless, the timings were useful for screening the responses for anomalies, that is, to eliminate the data for subjects who responded too quickly (and thus probably did not complete the experiment in a serious fashion) or those who responded too slowly (and thus were probably distracted while doing the experiment). WebExp automatically tests the response timings against upper and lower limits provided by the experimenter and excludes participants whose timings are anomalous. Further manual checks can be carried out on the response timings later on.

Apart from providing response timing, WebExp also offers a set of safeguards that are meant to ensure the authenticity of the participants taking part and exclude participants from participating more than once:

E-mail Address. Each participant has to provide his or her e-mail address. An automatic plausibility check is conducted on the address to ensure that it is syntactically valid. If the address is valid, then WebExp sends an e-mail to the address at the end of the experiment (the e-mail typically contains a message thanking the participant for taking part). If the e-mail is returned as undeliverable, the experimenter is effectively informed that a participant is likely to have used a fake identity and has the option of excluding that participant's responses from further analysis.

Personal Data. Before the experiment proper commences, each participant has to fill in a short questionnaire supplying name, age, sex, handedness, and language background. These data allow manual plausibility checks so that participants who provide implausible answers can be eliminated from the data set.

6 The WebExp software package is distributed free of charge for noncommercial purposes. Information on how to obtain the latest version is available at http://www.hcrc.ed.ac.uk/web_exp/. A central entry page for all experiments using WebExp can be found at http://www.language-experiments.org/. 
Responses. A manual inspection of the responses allows the experimenter to detect participants who have misunderstood the instructions or who have responded in an anomalous fashion (e.g., by giving the same response to every item).

Connection Data. The software also logs data related to the participant's Web connection. This includes the Internet address of his machine and the operating system and browser he uses. This information (in addition to the e-mail address) is valuable in detecting participants who take part more than once.

In addition to making it possible to administer experiments over the Web, WebExp can also be used in a conventional laboratory setting. In such a setting, WebExp has the advantage of being platform independent (as it is implemented in Java); that is, it will run on any computer that is connected to the Internet and runs a Web browser. Comparisons of experimental data obtained from Internet experiments (using WebExp) and their laboratory-based counterparts (Keller and Asudeh 2002; Keller and Alexopoulou 2001; Corley and Scheepers 2002) revealed high correlations between the two types of data sets; the comparisons also demonstrated that the same main effects are obtained from Web-based and laboratory-based experiments.

\section{Appendix B. Materials}

\section{B.1. Experiment 1}

The following is a list of the materials used in Experiment 1. The modulus is shown in (64). The verb-noun pairs and their selected interpretations (Interpr) are illustrated in Table 23. In addition, the table shows the mean ratings (Rtg) for each paraphrase according to the subjects' responses and their probability (Prob) according to the model.

$$
\text { David finished a course David finished writing a course }
$$

\section{B.2. Experiment 4}

The experimental item in (65) was presented as the modulus in Experiment 4 . The experimental materials are shown in Tables 24 and 25 .

hard substance a substance that is hard to alter 
Table 23

Materials for Experiment 1, with mean ratings and model probabilities.

\begin{tabular}{|c|c|c|c|c|c|c|c|c|c|}
\hline \multirow[b]{2}{*}{ Verb-noun } & \multicolumn{3}{|c|}{ High } & \multicolumn{3}{|c|}{ Medium } & \multicolumn{3}{|c|}{ Low } \\
\hline & Interpr & Rtg & Prob & Interpr & & Prob & Interpr & Rtg & Prob \\
\hline $\begin{array}{l}\text { attempt definition } \\
\text { attempt peak } \\
\text { attempt question } \\
\text { attempt smile } \\
\text { attempt walk }\end{array}$ & $\begin{array}{l}\text { analyze } \\
\text { climb } \\
\text { reply to } \\
\text { give } \\
\text { take }\end{array}$ & $\begin{array}{r}-0.087 \\
0.2646 \\
0.1416 \\
0.2490 \\
0.1807\end{array}$ & $\begin{array}{l}-21.44 \\
-20.22 \\
-18.96 \\
-19.43 \\
-19.81\end{array}$ & $\begin{array}{l}\text { recall } \\
\text { claim } \\
\text { set } \\
\text { rehearse } \\
\text { schedule }\end{array}$ & $\begin{array}{l}-0.0338 \\
-0.0900 \\
-0.2666 \\
-0.1976 \\
-0.1649\end{array}$ & $\begin{array}{l}-22.84 \\
-23.53 \\
-21.63 \\
-22.21 \\
-22.71\end{array}$ & $\begin{array}{l}\text { support } \\
\text { include } \\
\text { stick to } \\
\text { look at } \\
\text { lead }\end{array}$ & $\begin{array}{l}-0.1571 \\
-0.4450 \\
-0.3168 \\
-0.4722 \\
-0.1863\end{array}$ & $\begin{array}{l}-23.87 \\
-24.85 \\
-22.55 \\
-23.74 \\
-23.85\end{array}$ \\
\hline $\begin{array}{l}\text { egin game } \\
\text { begin photograph } \\
\text { begin production } \\
\text { begin test } \\
\text { begin theory }\end{array}$ & $\begin{array}{l}\text { play } \\
\text { develop } \\
\text { organize } \\
\text { take } \\
\text { formulate }\end{array}$ & $\begin{array}{l}0.2798 \\
0.0816 \\
0.0502 \\
0.2699 \\
0.2142\end{array}$ & $\begin{array}{l}-15.11 \\
-21.11 \\
-19.09 \\
-17.97 \\
-18.28\end{array}$ & $\begin{array}{l}\text { modify } \\
\text { test } \\
\text { influence } \\
\text { examine } \\
\text { present }\end{array}$ & $\begin{array}{r}-0.3403 \\
-0.2147 \\
-0.2329 \\
-0.1424 \\
0.1314\end{array}$ & $\begin{array}{l}-21.52 \\
-22.49 \\
-21.98 \\
-21.78 \\
-21.54\end{array}$ & $\begin{array}{l}\text { comman } \\
\text { spot } \\
\text { tax } \\
\text { assist } \\
\text { assess }\end{array}$ & $\begin{array}{l}-0.2415 \\
-0.4054 \\
-0.4367 \\
-0.3440 \\
-0.1356\end{array}$ & $\begin{array}{l}-23.46 \\
-23.69 \\
-22.78 \\
-24.11 \\
-22.40\end{array}$ \\
\hline $\begin{array}{l}\text { ljoy book } \\
\text { ijoy city } \\
\text { ijoy concert } \\
\text { hjoy dish } \\
\text { ijoy story }\end{array}$ & $\begin{array}{l}\text { read } \\
\text { live in } \\
\text { listen to } \\
\text { cook } \\
\text { write }\end{array}$ & $\begin{array}{r}0.2891 \\
0.2028 \\
0.2779 \\
-0.1223 \\
-0.0731\end{array}$ & $\begin{array}{l}-16.48 \\
-20.77 \\
-20.91 \\
-20.21 \\
-19.08\end{array}$ & $\begin{array}{l}\text { discuss } \\
\text { come to } \\
\text { throw } \\
\text { choose } \\
\text { learn }\end{array}$ & $\begin{array}{r}-0.1515 \\
0.1842 \\
-0.2442 \\
-0.2373 \\
-0.0887\end{array}$ & $\begin{array}{l}-23.33 \\
-23.50 \\
-23.61 \\
-24.61 \\
-23.50\end{array}$ & $\begin{array}{l}\text { build } \\
\text { cut } \\
\text { make } \\
\text { bring } \\
\text { choose }\end{array}$ & $\begin{array}{l}-0.5404 \\
-0.6957 \\
-0.2571 \\
-0.3385 \\
-0.2607\end{array}$ & $\begin{array}{l}-25.52 \\
-24.67 \\
-24.97 \\
-25.33 \\
-24.61\end{array}$ \\
\hline $\begin{array}{l}\text { expect order } \\
\text { expect poetry } \\
\text { expect reply } \\
\text { expect reward } \\
\text { expect supper }\end{array}$ & $\begin{array}{l}\text { hear } \\
\text { see } \\
\text { get } \\
\text { collect } \\
\text { eat }\end{array}$ & & $\begin{array}{l}-20.29 \\
-20.43 \\
-20.23 \\
-21.91 \\
-21.27\end{array}$ & $\begin{array}{l}\text { read } \\
\text { learn } \\
\text { listen to } \\
\text { claim } \\
\text { start }\end{array}$ & $\begin{array}{r}0.0628 \\
-0.0601 \\
0.1178 \\
0.1950 \\
0.0285\end{array}$ & $\begin{array}{l}-22.92 \\
-22.81 \\
-23.48 \\
-23.13 \\
-23.20\end{array}$ & $\begin{array}{l}\text { prepare } \\
\text { prove } \\
\text { share } \\
\text { extend } \\
\text { seek }\end{array}$ & $\begin{array}{l}-0.3634 \\
-0.4985 \\
-0.2725 \\
-0.3743 \\
-0.3526\end{array}$ & $\begin{array}{l}-23.25 \\
-25.00 \\
-23.77 \\
-23.52 \\
-23.91\end{array}$ \\
\hline $\begin{array}{l}\text { finish gig } \\
\text { finish novel } \\
\text { finish project } \\
\text { finish room } \\
\text { finish video }\end{array}$ & $\begin{array}{l}\text { play } \\
\text { translate } \\
\text { work on } \\
\text { wallpaper } \\
\text { watch }\end{array}$ & & $\begin{array}{l}-20.34 \\
-21.81 \\
-18.79 \\
-19.07 \\
-22.37 \\
\end{array}$ & $\begin{array}{l}\text { plan } \\
\text { examine } \\
\text { study } \\
\text { construct } \\
\text { analyze }\end{array}$ & $\begin{array}{r}-0.1780 \\
-0.1323 \\
0.0679 \\
0.0444 \\
-0.0482\end{array}$ & $\begin{array}{l}-24.47 \\
-24.01 \\
-24.31 \\
-22.48 \\
-24.33\end{array}$ & $\begin{array}{l}\text { use } \\
\text { take } \\
\text { sell } \\
\text { show } \\
\text { describe }\end{array}$ & $\begin{array}{l}-0.5341 \\
-0.4375 \\
-0.1692 \\
-0.6305 \\
-0.1718\end{array}$ & $\begin{array}{l}-25.69 \\
-25.53 \\
-25.05 \\
-24.59 \\
-25.26\end{array}$ \\
\hline $\begin{array}{l}\text { postpone bill } \\
\text { postpone decision } \\
\text { postpone payment } \\
\text { postpone question } \\
\text { postpone trial }\end{array}$ & $\begin{array}{l}\text { debate } \\
\text { make } \\
\text { make } \\
\text { hear } \\
\text { go to }\end{array}$ & $\begin{array}{l}0.0401 \\
0.3297 \\
0.2745 \\
0.1974 \\
0.1110\end{array}$ & $\begin{array}{l}-22.38 \\
-20.38 \\
-21.85 \\
-23.45 \\
-23.49 \\
\end{array}$ & & $\begin{array}{r}-0.2028 \\
-0.1087 \\
0.0166 \\
-0.0795 \\
-0.1425 \\
\end{array}$ & $\begin{array}{l}-25.36 \\
-24.03 \\
-23.21 \\
-24.70 \\
-25.50 \\
\end{array}$ & & $\begin{array}{r}-0.6238 \\
-0.4984 \\
-0.4675 \\
-0.5383 \\
0.0336 \\
\end{array}$ & $\begin{array}{l}-27.92 \\
-25.56 \\
-25.91 \\
-24.94 \\
-25.75 \\
\end{array}$ \\
\hline $\begin{array}{l}\text { prefer bike } \\
\text { prefer film } \\
\text { prefer gas } \\
\text { prefer people } \\
\text { prefer river }\end{array}$ & $\begin{array}{l}\text { ride } \\
\text { go to } \\
\text { use } \\
\text { talk with } \\
\text { swim in }\end{array}$ & & $\begin{array}{l}-20.64 \\
-21.63 \\
-20.28 \\
-20.52 \\
-19.53\end{array}$ & $\begin{array}{l}\text { mount } \\
\text { develop } \\
\text { measure } \\
\text { sit with } \\
\text { sail }\end{array}$ & $\begin{array}{r}-0.1617 \\
-0.1861 \\
-0.2903 \\
0.0283 \\
0.0433\end{array}$ & $\begin{array}{l}-22.95 \\
-23.07 \\
-23.88 \\
-22.75 \\
-22.93 \\
\end{array}$ & $\begin{array}{l}\text { go for } \\
\text { identify } \\
\text { encourage } \\
\text { discover } \\
\text { marry }\end{array}$ & $\begin{array}{l}-0.2119 \\
-0.4657 \\
-0.5074 \\
-0.2687 \\
-0.7269\end{array}$ & $\begin{array}{l}-24.49 \\
-24.69 \\
-25.33 \\
-25.26 \\
-24.13\end{array}$ \\
\hline $\begin{array}{l}\text { resist argument } \\
\text { resist invitation } \\
\text { resist pressure } \\
\text { resist proposal } \\
\text { resist song } \\
\end{array}$ & $\begin{array}{l}\text { contest } \\
\text { accept } \\
\text { take } \\
\text { work on } \\
\text { whistle }\end{array}$ & $\begin{array}{r}-0.0126 \\
0.2497 \\
-0.1481 \\
-0.0597 \\
-0.0013\end{array}$ & $\begin{array}{l}-22.66 \\
-21.56 \\
-22.67 \\
-23.56 \\
-22.11 \\
\end{array}$ & $\begin{array}{l}\text { continue } \\
\text { leave } \\
\text { make } \\
\text { take on } \\
\text { start }\end{array}$ & $\begin{array}{r}0.0224 \\
-0.3620 \\
-0.3602 \\
0.1286 \\
-0.1551 \\
\end{array}$ & $\begin{array}{l}-24.43 \\
-25.10 \\
-24.98 \\
-24.50 \\
-24.47 \\
\end{array}$ & $\begin{array}{l}\text { draw } \\
\text { offer } \\
\text { see } \\
\text { call } \\
\text { hold } \\
\end{array}$ & $\begin{array}{l}-0.4551 \\
-0.5301 \\
-0.4382 \\
-0.2906 \\
-0.5691 \\
\end{array}$ & $\begin{array}{l}-25.51 \\
-26.29 \\
-25.22 \\
-25.99 \\
-26.50 \\
\end{array}$ \\
\hline $\begin{array}{l}\text { start experiment } \\
\text { start letter } \\
\text { start treatment }\end{array}$ & $\begin{array}{l}\text { implement } \\
\text { write } \\
\text { receive }\end{array}$ & & $\begin{array}{l}-21.57 \\
-15.59 \\
-19.53\end{array}$ & & $\begin{array}{r}0.0184 \\
-0.0877 \\
0.1933\end{array}$ & $\begin{array}{l}-22.70 \\
-22.70 \\
-22.45\end{array}$ & & & $\begin{array}{l}-24.09 \\
-24.50 \\
-24.01\end{array}$ \\
\hline $\begin{array}{l}\text { survive course } \\
\text { survive journey } \\
\text { survive problem } \\
\text { survive scandal } \\
\text { survive wound }\end{array}$ & $\begin{array}{l}\text { give } \\
\text { make } \\
\text { create } \\
\text { experience } \\
\text { receive }\end{array}$ & $\begin{array}{r}0.0164 \\
0.2719 \\
-0.2697 \\
0.2200 \\
0.2012\end{array}$ & $\begin{array}{l}-22.87 \\
-22.31 \\
-21.12 \\
-24.61 \\
-24.49\end{array}$ & $\begin{array}{l}\text { make } \\
\text { take } \\
\text { indicate } \\
\text { create } \\
\text { produce }\end{array}$ & $\begin{array}{r}-0.1426 \\
0.2324 \\
-0.3267 \\
-0.2115 \\
-0.4361\end{array}$ & $\begin{array}{l}-24.48 \\
-24.43 \\
-23.01 \\
-26.02 \\
-26.32 \\
\end{array}$ & $\begin{array}{l}\text { write } \\
\text { claim } \\
\text { confirm } \\
\text { take } \\
\text { see }\end{array}$ & $\begin{array}{l}-0.1458 \\
-0.5388 \\
-0.3625 \\
-0.3068 \\
-0.2668\end{array}$ & $\begin{array}{l}-26.27 \\
-25.84 \\
-25.08 \\
-27.33 \\
-26.97\end{array}$ \\
\hline $\begin{array}{l}\text { try drug } \\
\text { try light } \\
\text { try shampoo } \\
\text { try sport } \\
\text { try vegetable }\end{array}$ & $\begin{array}{l}\text { take } \\
\text { turn } \\
\text { use } \\
\text { get into } \\
\text { eat }\end{array}$ & & $\begin{array}{l}-17.81 \\
-18.10 \\
-20.09 \\
-19.65 \\
-19.64 \\
\end{array}$ & $\begin{array}{l}\text { grow } \\
\text { reach for } \\
\text { pack } \\
\text { encourag } \\
\text { chop }\end{array}$ & $\begin{array}{l}-0.2516 \\
-0.1163 \\
-0.3496 \\
-0.2378 \\
-0.0780\end{array}$ & $\begin{array}{l}-22.09 \\
-21.23 \\
-21.56 \\
-21.09 \\
-21.38 \\
\end{array}$ & $\begin{array}{l}\text { hate } \\
\text { come with } \\
\text { like } \\
\text { consider } \\
\text { compare }\end{array}$ & $\begin{array}{l}-0.4777 \\
-0.5310 \\
-0.2581 \\
-0.2223 \\
-0.3248\end{array}$ & $\begin{array}{l}-23.88 \\
-23.93 \\
-24.56 \\
-22.82 \\
-22.38\end{array}$ \\
\hline $\begin{array}{l}\text { want bed } \\
\text { want hat } \\
\text { want man } \\
\text { want money } \\
\text { want program }\end{array}$ & $\begin{array}{l}\text { lay on } \\
\text { buy } \\
\text { marry } \\
\text { make } \\
\text { produce }\end{array}$ & $\begin{array}{r}0.1154 \\
0.2560 \\
0.0826 \\
0.1578 \\
-0.1980\end{array}$ & $\begin{array}{l}-19.17 \\
-17.84 \\
-15.50 \\
-15.16 \\
-18.86\end{array}$ & $\begin{array}{l}\text { reserve } \\
\text { examine } \\
\text { torment } \\
\text { handle } \\
\text { teach }\end{array}$ & $\begin{array}{r}0.0369 \\
-0.2127 \\
-0.2764 \\
-0.0929 \\
-0.1743\end{array}$ & $\begin{array}{l}-21.24 \\
-21.56 \\
-20.99 \\
-20.91 \\
-20.94\end{array}$ & $\begin{array}{l}\text { settle in } \\
\text { land on } \\
\text { assess } \\
\text { represent } \\
\text { hate }\end{array}$ & $\begin{array}{r}0.0015 \\
-0.6379 \\
-0.2510 \\
-0.4031 \\
-0.6788\end{array}$ & $\begin{array}{l}-22.26 \\
-22.38 \\
-22.22 \\
-22.92 \\
-22.63\end{array}$ \\
\hline
\end{tabular}


Table 24

Materials for Experiment 4, with mean ratings (object interpretations).

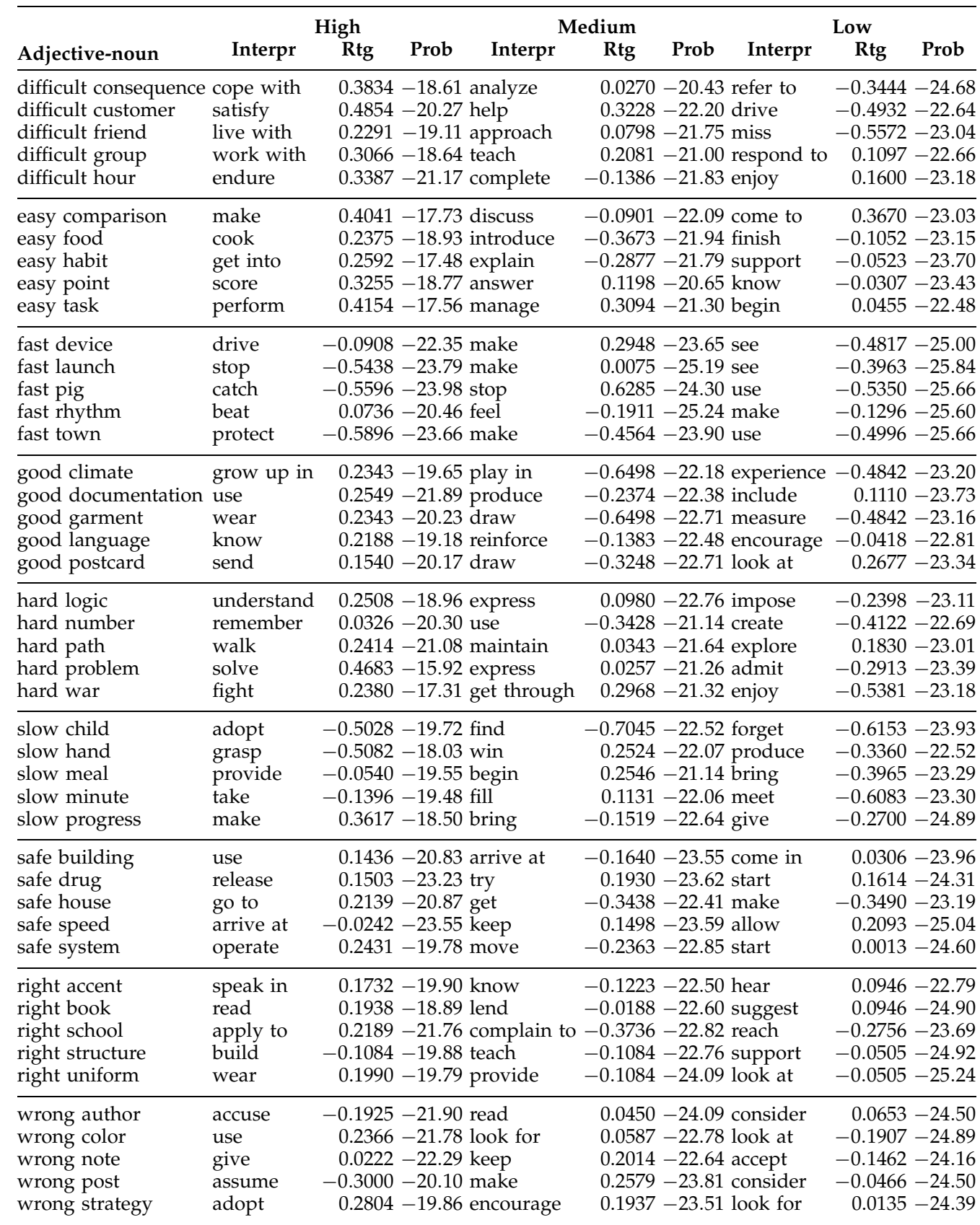


Table 25

Materials for Experiment 4, with mean ratings (subject interpretations).

\begin{tabular}{|c|c|c|c|c|c|c|c|c|c|}
\hline \multirow{2}{*}{ Adjective-noun } & \multicolumn{3}{|c|}{ High } & \multicolumn{3}{|c|}{ Medium } & \multicolumn{3}{|c|}{ Low } \\
\hline & Interpr & Rtg & Prob & Interpr & Rtg & Prob & Interpr & Rtg & Prob \\
\hline difficult customer & buy & -0.2682 & -20.19 & pick & -0.2050 & -22.58 & begin & -0.3560 & -24.83 \\
\hline & expla & -0.4658 & -20.16 & & .5274 & -21.75 & enjoy & -0.4711 & \\
\hline 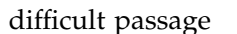 & read & 0.1668 & -20.46 & speak & -0.3600 & -22.62 & appear & -0.4030 & -23.78 \\
\hline d & read & 0.1052 & -20.30 & survive & -0.5080 & -22.28 & continue & -0.1006 & -23.89 \\
\hline difficult & break & -0.3047 & -19.80 & create & -0.2412 & -22.81 & start & -0.3661 & -23.50 \\
\hline asy car & start & -0.1652 & -21.01 & iove & -0.2401 & -21.42 & close & -0.5750 & -23.94 \\
\hline sy change & cur & 0.1999 & -20.32 & & 0.2332 & -21.57 & sit & -0.0932 & -23.27 \\
\hline od & ok & 0.0443 & -20.28 & change & -0.6046 & -22.25 & form & -0.3918 & -22.96 \\
\hline$y$ habit & develop & 0.1099 & -21.43 & start & 0.1156 & -23.21 & appear & -0.3490 & -24.70 \\
\hline easy task & fit & -0.3882 & -20.77 & end & -0.0982 & -22.90 & continue & 0.0474 & -24.11 \\
\hline fast device & go & 0.2638 & -22.80 & $\mathrm{CO}$ & -0.3652 & -23.91 & add & -0.2219 & -24.68 \\
\hline & $\mathrm{n}$ & 0.4594 & -20.78 & work & 0.0025 & -22.98 & add & .5901 & -24.64 \\
\hline t lady & walk & -0.0261 & -22.31 & work & -0.0716 & -23.90 & see & -0.4816 & -25.15 \\
\hline & run & 0.2081 & -22.57 & come & -0.1807 & -23.91 & get & -0.2764 & -24.75 \\
\hline fast town & grow & -0.3601 & -18.66 & spread & -0.3289 & -22.84 & sell & -0.3462 & -24.33 \\
\hline good ad & read & 0.1248 & -22.39 & sell & 0.2154 & -22.72 & run & -0.0832 & -22.78 \\
\hline imate & nange & -0.3748 & -21.30 & imprc & 3312 & -22 & begin & -0.4093 & -23.36 \\
\hline $\mathrm{sg}$ & look & -0.0581 & -21 & deve & 457 & -22 & appe & 0.1149 & -24.01 \\
\hline ght & work & -0.0022 & -19.42 & & -0.2023 & -21 & incre & 4349 & -23.82 \\
\hline & run & 0.0787 & -21.07 & continue & -0.0798 & -22.79 & die & -0.6569 & -23.79 \\
\hline hard fish & bite & -0.3583 & -20.39 & pull & -0.2579 & -22.53 & appear & -0.2568 & -22.79 \\
\hline & $\mathrm{g}$ & -0.1211 & -21.90 & & 4533 & -22 & go & 388 & -24.59 \\
\hline substance & keep & 0.0227 & -21.28 & rema & 0.0978 & -22.9 & seem & 0.1971 & -23.03 \\
\hline rd toilet & flush & -0.1796 & -20.46 & look & -0.3465 & -23.77 & start & -0.6835 & -24.60 \\
\hline hard war & break out & -0.4969 & -16.94 & grow & -0.2792 & -22.21 & increase & -0.2602 & -23.60 \\
\hline fe building & approach & -0.6815 & -22.87 & stay & 0.0852 & -23.09 & start & -0.5152 & -23.53 \\
\hline & $\mathrm{me}$ & 302 & -19 & & 5562 & -22 & try & 126 & 22.45 \\
\hline & & -0.5434 & -21.23 & igno & -0.6673 & -21.8 & agre & -0.6509 & -23.16 \\
\hline & & -0.3116 & -16.26 & leave & -0.6136 & -20.24 & rema & 0.1267 & -22.87 \\
\hline safe system & operate & 0.3697 & -19.92 & continue & 0.0374 & -21.48 & think & -0.4845 & -22.90 \\
\hline our hild & & 0.1485 & -22.66 & & 0.1556 & -23.21 & & -0.0256 & -24.60 \\
\hline & mor & .0738 & & & 0039 & & & 346 & -25.56 \\
\hline & & 0.1237 & -20 & & -0.1474 & -21.47 & becor & -0.2802 & -23.17 \\
\hline inute & pass & 0.2717 & -23.17 & start & -0.4423 & -23.49 & win & -0.6709 & -24.64 \\
\hline & come & -0.0671 & -19.56 & follow & -0.3108 & -22.56 & seem & -0.2169 & -24.85 \\
\hline right accent & & -0.1727 & -18.54 & sound & 0.1928 & -21.14 & fall & -0.3926 & -22.76 \\
\hline & & -0.2429 & -19.50 & feel & -0.1027 & -21.74 & & -0.2195 & -23.61 \\
\hline t character & live & -0.2505 & -22.65 & set & -0.4063 & -23.14 & feel & -0.1651 & -23.92 \\
\hline eople & vote & -0.4541 & -21.70 & eat & -0.5921 & -22.81 & answer & -0.0992 & -24.68 \\
\hline & teach & 0.0159 & -20.91 & start & -0.3466 & -24.03 & stand & -0.3839 & -24.88 \\
\hline ono on & & -0.4348 & -20.59 & & -0.4128 & -22.96 & rea & -0.5542 & -24.50 \\
\hline wrong busine & think & -0.4018 & -23.15 & & & -23.85 & hope & -0.5608 & -24.18 \\
\hline wrong color & go & 0.1846 & -20.93 & show & -0.0819 & -23.54 & seem & 0.2869 & -24.18 \\
\hline wrong note & conclude & -0.2575 & -21.24 & show & -0.3480 & -23.87 & tell & -0.2732 & -24.45 \\
\hline wrong policy & encourage & -0.0401 & -21.71 & identify & -0.2167 & -23.56 & accept & 0.0183 & -23.76 \\
\hline
\end{tabular}




\section{Appendix C. Descriptive Statistics}

Table 26 displays the descriptive statistics for the model probabilities and the subject ratings for Experiments 1 and 4.

Table 26

Descriptives for model probabilities and subject ratings.

Rank Mean StdDev StdErr Min Max

Model probabilities, Experiment 1

\begin{tabular}{llllll}
\hline High & -21.62 & 2.59 & 0.26 & -24.62 & -15.11 \\
Medium & -22.65 & 2.19 & 0.22 & -26.32 & -20.90 \\
Low & -23.23 & 2.28 & 0.22 & -27.92 & -22.22 \\
\hline
\end{tabular}

Subject ratings, Experiment 1

\begin{tabular}{lrrrrr}
\hline High & 0.1449 & 0.2355 & 0.0309 & -0.2697 & 0.3297 \\
Medium & -0.1055 & 0.2447 & 0.0321 & -0.4361 & 0.2324 \\
Low & -0.3848 & 0.2728 & 0.0358 & -0.7269 & 0.0336 \\
\hline
\end{tabular}

Model probabilities, Experiment 4

\begin{tabular}{llllll}
\hline High & -20.49 & 1.71 & 0.18 & -23.99 & -15.93 \\
Medium & -22.62 & 0.99 & 0.10 & -25.24 & -20.24 \\
Low & -23.91 & 0.86 & 0.18 & -25.85 & -22.46 \\
\hline
\end{tabular}

Subject ratings, Experiment 4

\begin{tabular}{llllll}
\hline High & -0.0005 & 0.2974 & 0.0384 & -0.68 & 0.49 \\
Medium & -0.1754 & 0.3284 & 0.0424 & -0.70 & 0.31 \\
Low & -0.2298 & 0.3279 & 0.0423 & -0.68 & 0.37
\end{tabular}

\section{Acknowledgments}

This work was supported by ESRC grant number R000237772 (Data Intensive Semantics and Pragmatics) and the DFG (Gottfried Wilhelm Leibniz Award to Manfred Pinkal). Alex Lascarides is supported by an ESRC research fellowship. Thanks to Brian McElree and Matt Traxler for making available to us the results of their norming study and to Ann Copestake, Frank Keller, Scott McDonald, Manfred Pinkal, Owen Rambow, and three anonymous reviewers for valuable comments.

\section{References}

Abney, Steve. 1996. Partial parsing via finite-state cascades. In John Carroll, editor, Workshop on Robust Parsing, Prague. European Summer School in Logic, Language and Information, pages 8-15. Apresjan, J. D. 1973. Regular polysemy. Linguistics, 142:5-32.

Bard, Ellen Gurman, Dan Robertson, and Antonella Sorace. 1996. Magnitude estimation of linguistic acceptability. Language, 72(1):32-68.

Barzilay, Regina and Kathy McKeown. 2001. Extracting paraphrases from a parallel corpus. In Proceedings of the 39th Annual Meeting of the Association for Computational Linguistics, Toulouse, France, pages 50-57.

Bouaud, Jacques, Bruno Bachimont, and Pierre Zweigenbaum. 1996. Processing metonymy: A domain-model heuristic graph traversal approach. In Proceedings of the 16th International Conference on Computational Linguistics, Copenhagen, Denmark, pages 137-142.

Briscoe, Ted, Ann Copestake, and Bran Boguraev. 1990. Enjoy the paper: Lexical semantics via lexicology. In Proceedings of the 13th International Conference on Computational Linguistics, Helsinki, Finland, pages 42-47.

Burnage, Gavin. 1990. Celex-A guide for users. Technical report, Centre for Lexical Information, University of Nijmegen, Nijmegen, the Netherlands.

Burnard, Lou. 1995. The Users Reference Guide for the British National Corpus. British 
National Corpus Consortium, Oxford University Computing Service, Oxford, U.K.

Clark, Stephen and David Weir. 2001. Class-based probability estimation using a semantic hierarchy. In Proceedings of the Second Conference of the North American Chapter of the Association for Computational Linguistics, Pittsburgh, Pennsylvania.

Collins, Michael and James Brooks. 1995. Prepositional phrase attachment through a backed-off model. In David Yarowsky and Kenneth W. Church, editors, Proceedings of the Third Workshop on Very Large Corpora, Cambridge, Massachusetts, pages $27-38$.

Copestake, A. 2001. The semi-generative lexicon: Limits on lexical productivity. In Proceedings of the First International Workshop on Generative Approaches to the Lexicon, Geneva.

Copestake, A. and E. J. Briscoe. 1995. Semi-productive polysemy and sense extension. Journal of Semantics, 12(1):15-67.

Copestake,A. and A. Lascarides. 1997. Integrating symbolic and statistical representations: The lexicon pragmatics interface. In Proceedings of the 35th Annual Meeting of the Association for Computational Linguistics and the Eighth Meeting of the European Chapter for the Association for Computational Linguistics, Madrid, pages 136-143.

Corley, Martin and Christoph Scheepers. 2002. Syntactic priming in English: Evidence from response latencies. Psychonomic Bulletin and Review, 9(1):126-131.

Cowart, Wayne. 1997. Experimental Syntax: Applying Objective Methods to Sentence Judgments. Sage, Thousand Oaks, California.

Daelemans, Walter, Antal van den Bosch, and Jakub Zavrel. 1999. Forgetting exceptions is harmful in language learning. Machine Learning, 34(1-3):11-43.

Fass, Dan. 1991. met*: A method for discriminating metonymy and metaphor by computer. Computational Linguistics, 17(1):49-90.

Godard, Danièle and Jacques Jayez. 1993. Towards a proper treatment of coercion phenomena. In Proceedings of the 31st Annual Meeting of the Association for Computational Linguistics, Columbus, Ohio, pages 168-177.

Hobbs, Jerry R., Martin Stickel, Douglas Appelt, and Paul Martin. 1993. Interpretation as abduction. Artificial Intelligence, 63(1-2):69-142.

Inverson, Eric and Stephen Helmreich. 1992. An integrated approach to non-literal phrase interpretation. Computational Intelligence, 8(3):477-493.

Keller, Frank and Theodora Alexopoulou. 2001. Phonology competes with syntax: Experimental evidence for the interaction of word order and accent placement in the realization of information structure. Cognition, 79(3):301-371.

Keller, Frank and Ash Asudeh. 2002. Probabilistic learning algorithms and optimality theory. Linguistic Inquiry, 33(2):225-244.

Keller, Frank, Martin Corley, and Christoph Scheepers. 2001. Conducting psycholinguistic experiments over the World Wide Web. Unpublished manuscript, University of Edinburgh, Edinburgh, U.K., and Saarland University, Saarbruecken, Germany.

Lakoff, George and Mark Johnson. 1980. Metaphors We Live By. University of Chicago Press, Chicago.

Lapata, Maria, Frank Keller, and Scott McDonald. 2001. Evaluating smoothing algorithms against plausibility judgments. In Proceedings of the 39th Annual Meeting of the Association for Computational Linguistics, Toulouse, France, pages 346-353.

Lascarides, Alex and Ann Copestake. 1998. Pragmatics and word meaning. Journal of Linguistics, 34(2):387-414.

Leech, Geoffrey, Roger Garside, and Michael Bryant. 1994. The tagging of the British national corpus. In Proceedings of the 15th International Conference on Computational Linguistics, Kyoto, Japan, pages 622-628.

Levin, Beth. 1993. English Verb Classes and Alternations: A Preliminary Investigation. University of Chicago Press, Chicago.

Li, Hang and Naoki Abe. 1998. Generalizing case frames using a thesaurus and the MDL principle. Computational Linguistics, 24(2):217-244.

Light, Marc. 1996. Morphological Cues for Lexical Semantics. Ph.D. thesis, University of Rochester.

Lodge, Milton. 1981. Magnitude Scaling: Quantitative Measurement of Opinions. Sage, Beverly Hills, California.

Macleod, Catherine, Ralph Grishman, Adam Meyers, Leslie Barrett, and Ruth Reeves. 1998. Comlex: A lexicon of nominalizations. In Proceedings of the Eighth International Congress of the European Association for Lexicography, Liège, Belgium, pages 187-193.

Markert, Katja and Udo Hahn. 1997. On the interaction of metonymies and anaphora. In Proceedings of the 15th International Joint Conference on Artificial Intelligence, Nagoya, Japan, pages 1010-1015. 
McCarthy, Diana. 2000. Using semantic preferences to identify verbal participation in role switching alternations. In Proceedings of the First Conference of the North American Chapter of the Association for Computational Linguistics, Seattle, Washington, pages 256-263.

McElree, Brian, Matthew J. Traxler, Martin J. Pickering, Rachel E. Seely, and Ray Jackendoff. 2001. Reading time evidence for enriched composition. Cognition, (78)1:17-25.

Merlo, Paola and Susanne Stevenson. 2001. Automatic verb classification based on statistical distribution of argument structure. Computational Linguistics, 27(3):373-408.

Miller, George A., Richard Beckwith, Christiane Fellbaum, Derek Gross, and Katherine J. Miller. 1990. Introduction to WordNet: An on-line lexical database. International Journal of Lexicography, 3(4):235-244.

Nunberg, Geoffrey. 1995. Transfers of meaning. Journal of Semantics, 12(1):109-132.

Pustejovsky, James. 1991. The generative lexicon. Computational Linguistics, 17(4):409-441.

Pustejovsky, James. 1995. The Generative Lexicon. MIT Press, Cambridge.

Pustejovsky, James and Pierrette Bouillon. 1995. Logical polysemy and aspectual coercion. Journal of Semantics, 12:133-162.

Resnik, Philip. 1993. Selection and Information: A Class-Based Approach to Lexical Relationships. Ph.D. thesis, University of Pennsylvania, Philadelphia.

Resnik, Philip. 1999. Semantic similarity in a taxonomy: An information-based measure and its application to problems of ambiguity in natural language. Journal of Artificial Intelligence Research, 95-130.
Resnik, Philip and Mona Diab. 2000. Measuring verb similarity. In Lila R. Gleitman and Aravid K. Joshi, editors, Proceedings of the 22nd Annual Conference of the Cognitive Science Society. Erlbaum, Mahwah, New Jersey, pages 399-404.

Rooth, Mats, Stefan Riezler, Detlef Prescher, Glenn Carroll, and Franz Beil. 1999.

Inducing a semantically annotated lexicon via em-based clustering. In Proceedings of the 37th Annual Meeting of the Association for Computational Linguistics, College Park, Maryland, pages 104-111.

Schütze, Carson T. 1996. The Empirical Base of Linguistics: Grammaticality Judgments and Linguistic Methodology. University of Chicago Press, Chicago.

Siegel, Eric and Kathleen McKeown. 2000. Learning methods to combine linguistic indicators: Improving aspectual classification and revealing linguistic insights. Computational Linguistics, 26(4):595-629.

Stevens, S. Smith. 1975. Psychophysics: Introduction to Its Perceptual, Neural, and Social Prospects. Wiley, New York.

Utiyama, Masao, Masaki Murata, and Hitoshi Isahara. 2000. A statistical approach to the processing of metonymy. In Proceedings of the 18th International Conference on Computational Linguistics, Saarbrücken, Germany, pages 885-891.

Vendler, Zeno. 1968. Adjectives and Nominalizations. Mouton, The Hague, the Netherlands.

Verspoor, Cornelia Maria. 1997. ContextuallyDependent Lexical Semantics. Ph.D. thesis, University of Edinburgh, Edinburgh, U.K.

Weiss, Sholom M. and Casimir A. Kulikowski. 1991. Computer Systems that Learn: Classification and Prediction Methods from Statistics, Neural Nets, Machine Learning, and Expert Systems. Morgan Kaufmann, San Mateo, California. 